Research Article

\title{
Flexural Fatigue Life Reliability of Alkali-Activated Slag Concrete Freeze-Thaw Damage in Cold Areas
}

\author{
Bin Chen and Jun Wang \\ College of Civil Engineering, Northeast Forestry University, No. 26 Hexing Road, Harbin 150040, China \\ Correspondence should be addressed to Jun Wang; jun.w.619@nefu.edu.cn
}

Received 4 June 2021; Revised 8 July 2021; Accepted 12 August 2021; Published 27 August 2021

Academic Editor: Abílio De Jesus

Copyright ( 92021 Bin Chen and Jun Wang. This is an open access article distributed under the Creative Commons Attribution License, which permits unrestricted use, distribution, and reproduction in any medium, provided the original work is properly cited.

\begin{abstract}
Studying the application of alkali-activated slag concrete for roads in cold areas is of great significance for promoting and developing green building materials. In this study, the effect of freeze-thaw damage on the flexural fatigue properties of alkaliactivated slag concrete was studied and the fatigue life of alkali-activated slag concrete with various degrees of damage after freezethaw cycles was studied through a three-point flexural test. The results show that the flexural fatigue life decreases with freeze-thaw cycles from 0 to 150 times. Through a distribution fitting test and K-S test results, the flexural fatigue life followed both the twoparameter and three-parameter Weibull distributions. Between them, the three-parameter Weibull distribution fitting had a higher accuracy and better test results. The results of the reliability analysis show that the curves of alkali-activated slag concrete samples with various degrees of freeze-thaw damage for various failure probabilities have good correlation under different stresses, and the correlation correlations were greater than 0.81 . The flexural fatigue life of alkali-activated slag concrete samples with various degrees of freeze-thaw damage was more sensitive to freeze-thaw damage under high stresses. It is suggested that the fatigue design of alkali-activated slag concrete should consider the adverse effects of cold areas, and the reliability should be improved accordingly.
\end{abstract}

\section{Introduction}

Cement concrete is one of the most widely used materials for road and bridge building worldwide $[1,2]$. However, with the continuous development of the economy and the continuous expansion of infrastructure, the durability of cement concrete structures, including factors such as material degradation and decreased service life, has become an evident problem [3, 4]. Freeze-thaw damage is one of the important factors influencing the degradation of concrete structures in cold areas and seriously influences the durability of concrete structures $[5,6]$. Cement concrete in roads and bridges in severely cold areas is damaged by freeze-thaw cycles and subjected to frequent traffic loads, which deteriorate the performance of the concrete structure and thus greatly increase the construction and maintenance costs [7].

Alkali-activated slag concrete (AASC) is an environment-friendly building material that uses slag as a raw material in the role of a proper alkali excitation agent, which is mixed with an appropriate amount of water as well as sand and gravel to prepare concrete $[8,9]$. AASC has the characteristics and advantages of low-carbon environmental protection and industrial solid waste reuse, making it a building material with a bright future [10-12]. In addition, AASC has been confirmed to have high early strength and good resistance to high temperatures [13-15]. Some studies have also confirmed that compared with cement concrete, AASC has better frost $[16,17]$ and fatigue $[18,19]$ resistance. Therefore, the application and promotion of AASC in roads and bridges in cold areas has excellent potential and great research value.

At present, there are few studies on the interaction between freeze-thaw cycles and the fatigue behavior of AASC. Because the test periods for freeze-thaw failure and fatigue failure are quite different, it is difficult to use existing test equipment to conduct an experimental study and 
analysis of the coupling effect of the two failure mechanisms. Scholars have performed some research of AASC freezethaw damage and fatigue damage, respectively. Provis et al. [20] found that the frost resistance of alkali-activated gelled material is higher than that of cement-gelled material. Shahrajabian and Behfarnia [21] reported that an AASC mixed with $\mathrm{Al}_{2} \mathrm{O}_{3}$ exhibited improved frost resistance. $\mathrm{Ma}$ et al. [22] reported that AASC freeze-thaw damage was caused by damage to the aggregate and mortar interface area and that the increasing damage led to the final failure. However, the failure of AASC by fatigue mainly focuses on the study of damage mechanisms. Candido et al. [23] found that AASC had a higher fatigue life than that of cement concrete, and higher stress levels exhibited a more noticeable fatigue performance advantage. A study of the damage mechanism found that bonding between the AASC-gelled material and aggregate in concrete was stronger than that in cement concrete, which resulted in better fatigue performance for the AASC. Mohammadinia et al. [24] reported that aluminosilicate cementitious material in the hydration products of AASC had better bonding ability and ductility, which could absorb cyclic load stress and delay the development of cracks; thus, it has good flexural fatigue durability. In summary, research on the freeze-thaw and fatigue durability of AASC has mostly been conducted separately and has mainly focused on the damage mechanism. Therefore, it is necessary to study AASC under the combined effects of freeze-thaw cycles and fatigue damage, mainly focusing on the fatigue life of AASC under frost damage and the reliability probability distribution of fatigue life and fatigue life equation and providing a design and application reference and basis for AASC in road and bridge engineering.

Based on the abovementioned condition, the flexural fatigue life of AASC with various degrees of freeze-thaw damage was studied experimentally. The distribution of the flexural fatigue life of AASC with various freeze-thaw damage models under various stress levels is presented and discussed. The reliability probability of the flexural fatigue life of AASC subjected to freeze-thaw damage was obtained, and the flexural fatigue life equations of AASC with various degrees of freeze-thaw damage with various given failure probabilities were established, which provided a theoretical basis for the design and verification of fatigue performance of AASC.

\section{Materials and Methods}

2.1. Raw Materials and Mix Proportions. Slag is the granulated blast furnace slag of Yingkou Wukuang Zhongban Minmetals Group, with a specific surface area of $408 \mathrm{~m}^{2} / \mathrm{kg}$ and a density of $2.92 \mathrm{~g} / \mathrm{cm}^{3}$. The chemical composition of the slag is shown in Table 1 and meets the requirements of Grade S95 in GB/T 18046-2017 standard [25]. The alkali activator is a compound alkali activator mixed with solid sodium silicate and solid sodium aluminate. The solid sodium silicate is $\mathrm{Na}_{2} \mathrm{SiO}_{3} \cdot 9 \mathrm{H}_{2} \mathrm{O}$ (the content ratio of $\mathrm{Na}_{2} \mathrm{O}$ to $\mathrm{SiO}_{2}$ is $1.03 \pm 0.03$ ), and the solid sodium aluminate is the analytical pure grade. The mixed water is tap water. The fine aggregate
TABLE 1: Chemical composition of slag (\%).

\begin{tabular}{lcccccccc}
\hline Type & $\mathrm{CaO}$ & $\mathrm{SiO}_{2}$ & $\mathrm{MgO}$ & $\mathrm{Al}_{2} \mathrm{O}_{3}$ & $\mathrm{MnO}$ & $\mathrm{TiO}_{2}$ & $\mathrm{~S}$ & $\mathrm{FeO}$ \\
\hline Slag & 40.91 & 32.82 & 7.87 & 14.93 & 0.48 & 0.92 & 0.91 & 1.16 \\
\hline
\end{tabular}

is common river sand with an apparent density of $2620 \mathrm{~kg} / \mathrm{m}^{3}$, a bulk density of $1506 \mathrm{~kg} / \mathrm{m}^{3}$, and a mud content (by mass) of $0.5 \%$. The coarse aggregate is limestone gravel, $5 \sim 20 \mathrm{~mm}$ continuous grading; the apparent density is $2600 \mathrm{~kg} / \mathrm{m}^{3}$, the bulk density is $1600 \mathrm{~kg} / \mathrm{m}^{3}$, and the mud content (by mass) is $0.4 \%$.

In this test, the AASC mix ratio that had good performance in the previous research of this research group was adopted, as shown in Table 2. The alkali activator was a compound solid alkali activator with $5 \% \mathrm{Na}_{2} \mathrm{O}$ content $\left(\mathrm{NaAlO}_{2}\right.$ and $\mathrm{Na}_{2} \mathrm{SiO}_{3}$ were the compound solid alkali activators according to the $\mathrm{Na}_{2} \mathrm{O}$ content and mass ratio of $1: 4$ ).

2.2. Sample Preparation and Test Methods. A total of 81 AASC samples $(100 \mathrm{~mm} \times 100 \mathrm{~mm} \times 400 \mathrm{~mm})$ were prepared and formed according to the above mix ratio. After the samples were removed from the mold 1 day after forming, they were cured with plastic film under standard curing conditions for 6 months before the test, in order to reduce the influence of curing age on the fatigue property test results of AASC. Among them, 3 samples were tested for flexural fatigue strength to determine the characteristic values of fatigue loading. All samples adopted the same mix ratio and manufacturing technology and were cast in a single casting to reduce the dispersion of test results.

The freeze-thaw test was conducted according to the rapid frozen-thaw method in GB/T 50082-2019 standard [26]. The rapid frozen-thaw method was $1 \mathrm{~h}$ for freezing and $2 \mathrm{~h}$ for thawing. The freezing temperature was $-18 \pm 2^{\circ} \mathrm{C}$, and the thawing temperature was $5 \pm 2^{\circ} \mathrm{C}$. In this study, only liquid medium was used for freezing test. The fatigue test adopts the three-point flexural loading mode, as shown in Figure 1. All samples were loaded with nonintermittent sinusoidal wave, and the loading frequency was $5 \mathrm{~Hz}$, as shown in Figure 2. The cyclic loading was carried out in stress control mode, and Instron control system was adopted to provide stable control level. Four kinds of fatigue loads, namely, $0.70,0.75,0.80$, and 0.85 , were adopted for the stress level, and the low stress and high stress ratio was 0.1 .

In practical application, the periods of fatigue load and freeze-thaw cycle are different, and the transient effect of fatigue load and the stages of freeze-thaw cycle are taken into account. To investigate the effects of frost damage on the flexural fatigue performance of AASC, this study designed the test plan: first AASC samples underwent $0,50,100$, and 150 freeze-thaw cycles, carried out in accordance with the various stress levels, respectively, to determine fatigue loading, until the samples were damaged. The specific number and schedule of samples are shown in Table 3, where $N$ represents the number of samples, $n$ represents the number of freeze-thaw cycles, $S$ represents the maximum stress level, $R$ represents the stress ratio, and $P_{\max }$ represents 
TABLE 2: The mix proportion of AASC.

\begin{tabular}{ccccc}
\hline \multirow{2}{*}{ Type } & \multirow{2}{*}{ Water-to-binder ratio (\%) } & \multicolumn{3}{c}{ Mixture design $\left(\mathrm{kg} / \mathrm{m}^{3}\right)$} \\
& & Slag & Stand & Stone \\
\hline AASC & 0.35 & 460 & 716 & 1074 \\
\hline
\end{tabular}

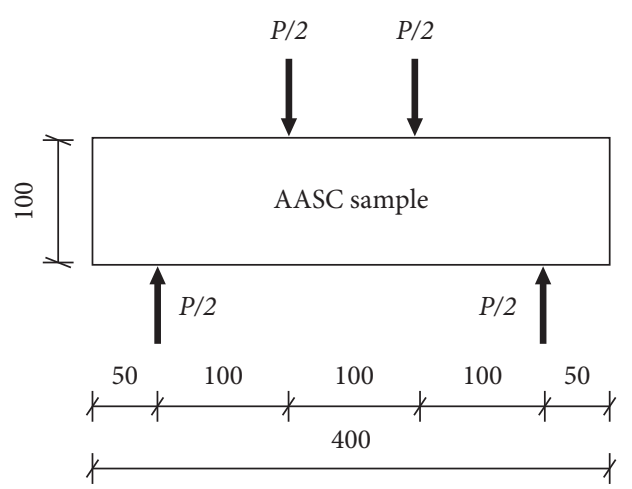

Figure 1: Schematic diagram of the flexural fatigue test.

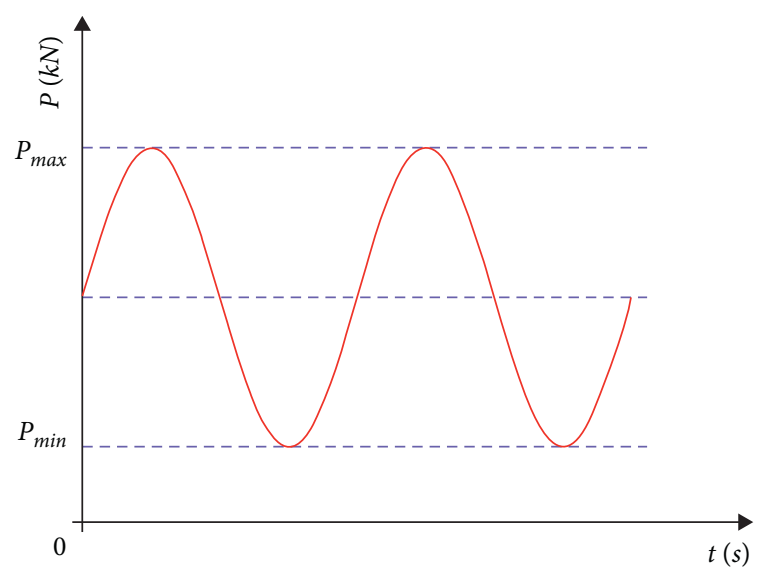

FIGURE 2: Fatigue loading parameter.

TABLE 3: Outline of samples preparation and experimental plan.

\begin{tabular}{lccccc}
\hline Code & $N$ & $n$ & $S$ & $R$ & $P_{\max }(\mathrm{kN})$ \\
\hline S0-1.00 & 3 & 0 & 1.00 & - & 19.41 \\
F0-0.70 & 6 & 0 & 0.70 & 0.10 & 13.59 \\
F0-0.75 & 6 & 0 & 0.75 & 0.10 & 14.56 \\
F0-0.80 & 6 & 0 & 0.80 & 0.10 & 15.53 \\
F0-0.85 & 6 & 0 & 0.85 & 0.10 & 16.50 \\
F50-0.70 & 6 & 50 & 0.70 & 0.10 & 13.59 \\
F50-0.75 & 6 & 50 & 0.75 & 0.10 & 14.56 \\
F50-0.80 & 6 & 50 & 0.80 & 0.10 & 15.53 \\
F100-0.70 & 6 & 100 & 0.70 & 0.10 & 13.59 \\
F100-0.75 & 6 & 100 & 0.75 & 0.10 & 14.56 \\
F100-0.80 & 6 & 100 & 0.80 & 0.10 & 15.53 \\
F150-0.70 & 6 & 150 & 0.70 & 0.10 & 13.59 \\
F150-0.75 & 6 & 150 & 0.75 & 0.10 & 14.56 \\
F150-0.80 & 6 & 150 & 0.80 & 0.10 & 15.53 \\
\hline
\end{tabular}

the maximum load. In the code of the sample number, $S$ represents the static load, $F$ represents the fatigue load, the second represents the number of freeze-thaw cycle times, and the third represents the loading stress level. For example, F-50-0.75 represents the fatigue loading of the sample at the stress level of 0.75 after 50 freeze-thaw cycle times. The flow chart of coupling experiment is shown in Figure 3.

\section{Test Results and Discussion}

3.1. Undamaged AASC. Twenty-four undamaged AASC samples were divided into four groups for flexural fatigue tests, with stress levels $(S)$ of $0.70,0.75,0.80$, and 0.85 for a total of four types of fatigue load levels. The flexural fatigue lives are shown in Table 4 , where $N_{i}$ represents the flexural fatigue life of the $i$-th sample under a specific stress level and $\bar{N}$ represents the average flexural fatigue life under a stress level.

The flexural fatigue test determined the corresponding flexural fatigue life by applying cyclic loads at various stress levels to the same samples. By studying the flexural fatigue test results, the $S-N$ curve, which describes the relationship between the stress level $S$ and the flexural fatigue life $N$, can be obtained; it is called the Wohler curve. Tepfers [27] and Oh [28] proposed and verified that the flexural fatigue behavior of concrete, represented by the $S-N$ curve describing the relationship between the stress level of concrete and the fatigue life, could be well expressed according to

$$
S=1-C(1-R) \lg N
$$

where $C$ is a coefficient, $R$ is the cyclic stress ratio, and $N$ is the fatigue life. Eq. (1) can be simplified to

$$
S=A+B \lg N
$$

where $A$ and $B$ are coefficients.

The values of constants $A$ and $B$ in eq. (2) can be obtained through regression analysis of the mean value of the test results in Table 4, and the $S$ - $\lg N$ curve of the AASC without freeze-thaw damage is shown in Figure 4. As shown in Figure 4, the correlation coefficient of the $S-\lg N$ curve is 0.9773 , indicating that the fitting curve has a good correlation and can effectively reflect the relationship between the stress level and flexural fatigue life.

Although the single-logarithmic $S$ - $\lg N$ curve can well reflect the relationship between the stress level and fatigue life, the $S-\lg N$ curve has clear limitations. The $S-\lg N$ curve cannot satisfy the boundary conditions at $N \longrightarrow \infty$ and $S \longrightarrow 0$; therefore, it is impossible to calculate the fatigue characteristics of AASC at low stress levels. Data show that the concrete structures used in road engineering are always in a state of low stress; therefore, it is of great significance to analyze the fatigue characteristics under low stress levels. However, it is unrealistic to reduce the stress level to $S \longrightarrow 0$ in a fatigue test. In addition, it will considerably increase the test time and cost if a lower stress level is selected for the test. Therefore, a double-logarithmic $\lg S-\lg N$ flexural fatigue equation is proposed, as shown in eq. (3). The $\lg S-\lg N$ equation is a relatively ideal form of the fatigue equation, which satisfies the boundary conditions at $N \longrightarrow \infty$ and $S \longrightarrow 0$ and fits the test results well [29]: 


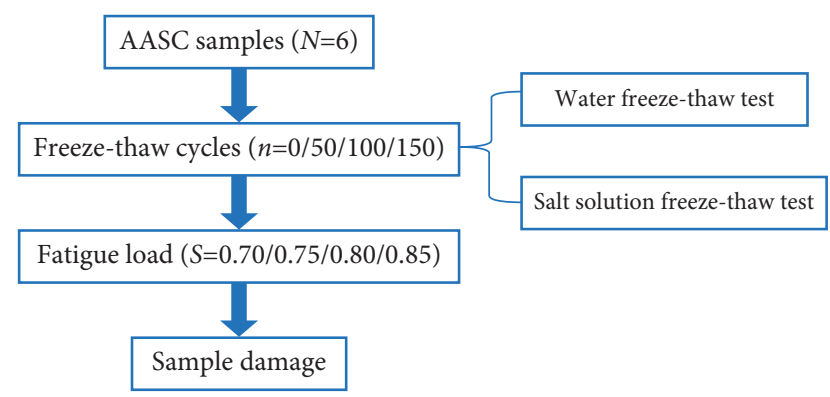

FIgURE 3: Flow chart of coupling experiment.

TABLE 4: Flexural fatigue lives of undamaged AASC samples.

\begin{tabular}{lccccccc}
\hline$S$ & $N_{1}$ & $N_{2}$ & $N_{3}$ & $N_{4}$ & $N_{5}$ & $N_{6}$ & $\bar{N}$ \\
\hline 0.70 & 196088 & 239314 & 247521 & 387625 & 465893 & 724824 & 376878 \\
0.75 & 65802 & 71825 & 97460 & 99129 & 105895 & 153130 & 98874 \\
0.80 & 3785 & 8142 & 17592 & 24321 & 28427 & 71825 & 25682 \\
0.85 & 649 & 993 & 1065 & 2325 & 2925 & 6483 & 2407 \\
\hline
\end{tabular}

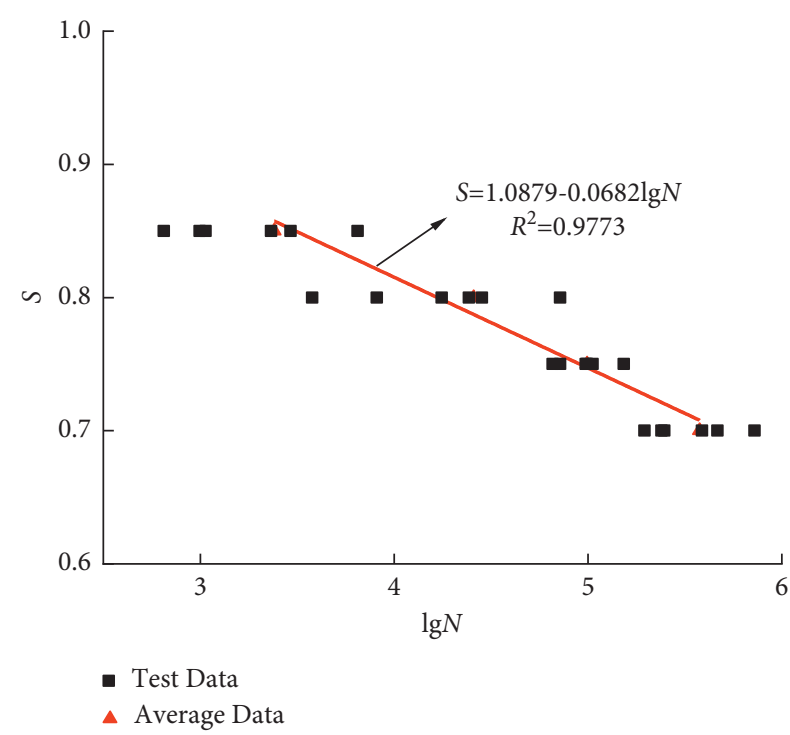

FIGURE 4: $S$ - $\lg (N)$ curve of the average fatigue life of undamaged AASC.

$$
\lg S=\lg a+b \lg N .
$$

The values of the constants $a$ and $b$ in eq. (3) can be obtained by regression analysis of the mean value of the test results in Table 4, and the $\lg S-\lg N$ curve of the undamaged AASC is shown in Figure 5, and the correlation coefficient of the $\lg S-\lg N$ curve is 0.9688 , indicating a good linear relationship between $\lg S$ and $\lg N$. In addition, the fatigue life characteristics of AASC at low stress levels can be evaluated using the $\lg S-\lg N$ curves.

3.2. Frost-Damaged AASC. The flexural fatigue tests of the undamaged AASC indicated that when the stress level was 0.85 , the flexural fatigue life was already small. Subjecting the AASC sample to various levels of frost damage will

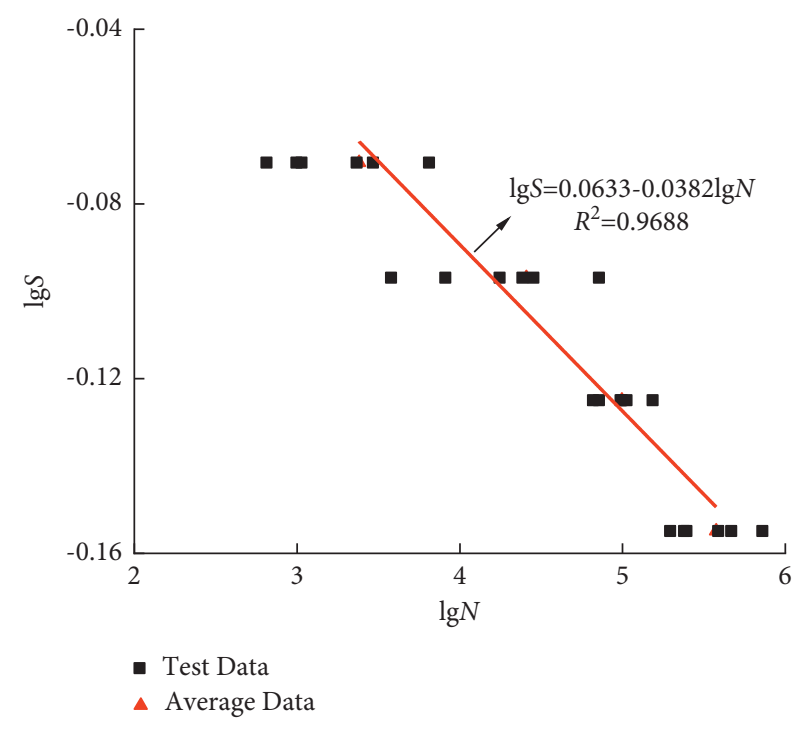

FIGURE 5: $\operatorname{lgS}-\operatorname{lgN}$ curve of the average fatigue life of undamaged AASC.

inevitably cause various degrees of damage inside the sample. Then, the flexural fatigue life will be even smaller under a 0.85 stress level. In addition, if concrete with a larger aggregate was considered, it would be difficult to obtain flexural fatigue life data from tests of the frost-damaged AASC at a 0.85 stress level. Therefore, only stress levels of $0.70,0.75$, and 0.80 were used for the flexural fatigue tests of the frost-damaged AASC.

The effects of freeze-thaw damage on the flexural fatigue life of AASC were examined. The effects of the freezing and thawing test and flexural fatigue test showed large differences, and the coupling experiment could not be conducted under the same test cycle. Hence, the test design was as follows: AASC first underwent various numbers of freezethaw cycles, and then, it underwent flexural fatigue tests at various stress levels. In the test design, it was ensured that the freeze-thaw damage had a significant impact on the flexural fatigue life of the AASC samples, but it was not so serious to make it impossible to measure the effective flexural fatigue life. Considering the above factors, AASC samples were designed to undergo 50, 100, and 150 freeze-thaw cycles before the flexural fatigue tests. They were divided into three groups according to the number freeze-thaw cycles with 18 samples in each group. Each group was subjected to flexural fatigue tests with stress levels of $0.70,0.75$, and 0.80. Each stress level had six samples for a total of 54 samples. To determine the influence of freeze-thaw damage on the flexural fatigue performance of the AASC samples, the loading stress levels at this stage were the same as the corresponding stress levels of the undamaged AASC samples. The flexural fatigue life test results of the frost-damaged AASC samples are listed in Table 5.

It can be seen from Table 5 that the flexural fatigue lives of the AASC samples with various degrees of freeze-thaw damage under the same stress level differed greatly. Under the same stress level, the flexural fatigue life of AASC decreased with increasing number of freeze-thaw cycles, 
TABLE 5: Flexural fatigue lives of frost-damaged AASC samples.

\begin{tabular}{ccccccccc}
\hline$n$ & $S$ & $N_{1}$ & $N_{2}$ & $N_{3}$ & $N_{4}$ & $N_{5}$ & $N_{6}$ \\
\hline \multirow{3}{*}{50} & 0.70 & 90865 & 164865 & 255060 & 290209 & 354585 & 584369 \\
& 0.75 & 18621 & 39435 & 56806 & 71796 & 132698 & 165615 & 80829 \\
& 0.80 & 4693 & 6685 & 9765 & 14065 & 37920 & 49871 & 20500 \\
\hline \multirow{3}{*}{100} & 0.70 & 61950 & 112750 & 184567 & 233058 & 261971 & 475382 \\
& 0.75 & 8526 & 18690 & 32795 & 69816 & 89830 & 157327 & 62831 \\
& 0.80 & 3448 & 4822 & 5769 & 8650 & 17423 & 25866 & 10996 \\
\hline \multirow{3}{*}{150} & 0.70 & 26378 & 58932 & 85668 & 103671 & 113590 & 260832 & 108179 \\
& 0.75 & 4986 & 6750 & 23954 & 25685 & 53581 & 55376 & 28389 \\
& 0.80 & 1005 & 1699 & 3025 & 4155 & 5562 & 12725 & 4695 \\
\hline
\end{tabular}

indicating that the fatigue stress of AASC decreases with increasing number of freeze-thaw cycles under the same fatigue strain. This means that the flexural fatigue strength of the AASC samples subjected to frost damage is smaller than that of the undamaged samples, indicating that frost damage intensifies the flexural fatigue failure of AASC. Frost damage causes the AASC sample to be repeatedly subjected to internal pressure and osmotic pressure, leading to loose flakes in the structure, and fatigue loading after the frost damage causes the AASC sample to be subjected to load stress, inflation pressure, and seepage pressure, a superposition of the set of adverse reactions, thus greatly reducing the flexural fatigue life of the AASC sample.

The values of the constants $A$ and $B$ in eq. (2) can be obtained by regression analysis of the mean values of the test results shown in Table 5. The flexural fatigue $S$ - $\lg N$ curves of the AASC samples with freeze-thaw damage are shown in Figure 6. As can be seen from Figure 6, the correlation coefficients of the $S-\lg N$ curves of the AASC samples with various degrees of freezing damage range from 0.9915 to 0.9996 . Therefore, $S$ and $\lg N$ have a good linear relationship, which effectively reflects the relationship between the stress level of the AASC samples and the flexural fatigue life after the corresponding frost damage.

To study the influence of frost damage on the fatigue characteristics of AASC under a low stress level, the doublelogarithmic flexural fatigue equation of the AASC sample after frost damage was established according to eq. (3). The values of the constants $a$ and $b$ in eq. (3) can be obtained by regression analysis of the average values of the test results shown in Table 5. The relationship of the frost-damaged AASC samples is shown in Figure 7.

Figure 7 shows that the flexural fatigue life curves of the AASC samples subjected to various degrees of frost damage are essentially parallel, but the curve moves to the left as the number of freeze-thaw cycles increases, indicating that the flexural fatigue life of AASC subjected to freeze-thaw damage decreases with increasing degree of frost damage. It also shows that the frost damage intensifies AASC flexural fatigue failure. In addition, the correlation coefficients of the $\lg S-\lg N$ curves for frost-damaged AASC in Figure 7 are between 0.9876 and 0.9984 , indicating a good linear relationship between $\lg S$ and $\lg N$. The fatigue life characteristics of AASC samples with various degrees of frost damage under low stress levels could be evaluated through the $\lg S-\lg N$ curves.

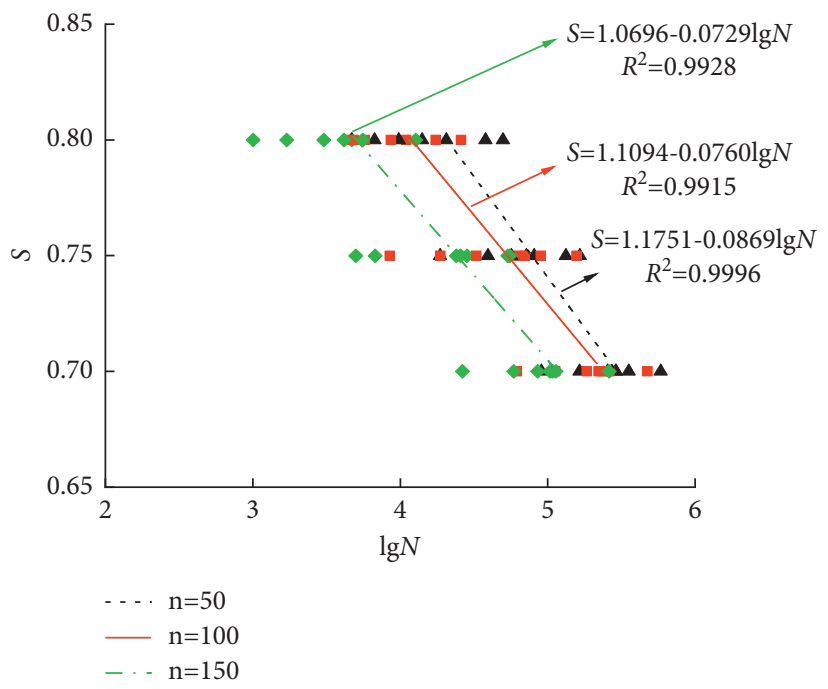

FIGURE 6: $S$ - $\lg N$ curves of the average fatigue life of frost-damaged AASC.

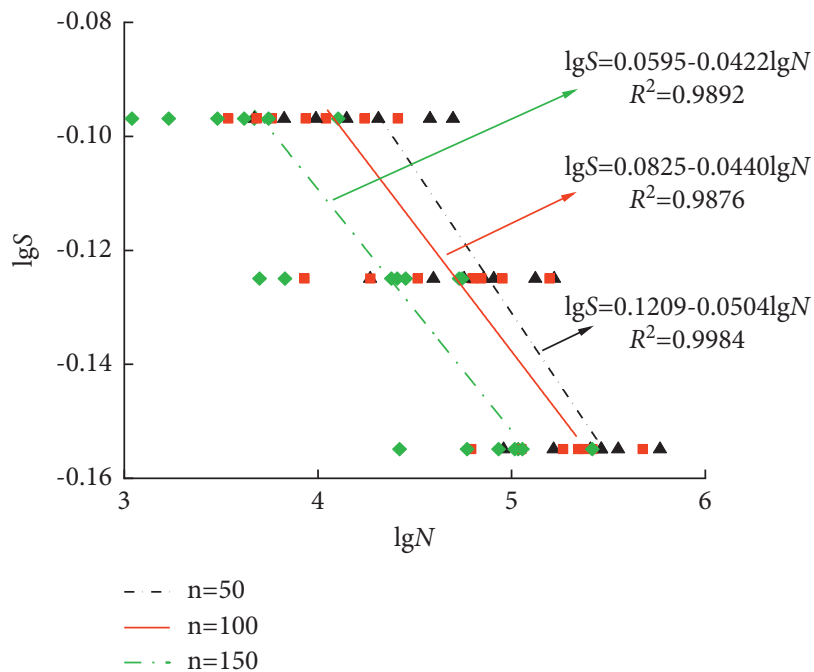

FIGURE 7: $\lg S-\lg N$ curves of the average fatigue life frost-damaged AASC.

\section{Flexural Fatigue Distribution Fitting}

The $S-\lg N$ and $\lg S-\lg N$ curves are obtained through regression analysis of the average flexural fatigue life; 
therefore, they are also called the mean flexural fatigue equation, which has a great value for theoretical study. However, its failure probability is close to $50 \%$, and it cannot be directly applied in engineering practice. AASC is a heterogeneous material, and fatigue loading has variability, which leads to discreteness in the flexural fatigue lives of AASC. However, an appropriate flexural fatigue strength must be determined during the design process to ensure sufficient fatigue resistance, and the fatigue strength must reflect the uncertainty in the fatigue behavior. Probabilistic reliability theory can effectively deal with this type of uncertainty, and a suitable mathematical model must be selected. Considering the actual service conditions of AASC in engineering applications, the Weibull model is the most suitable model to describe the distribution of the flexural fatigue life of AASC owing to the existence of the minimum safe life and the long fatigue life zone.

\subsection{Two-Parameter Weibull Distribution Theory of Flexural} Fatigue Life. Using the graphical algorithm and according to the definition of the Weibull probability distribution function, the two-parameter Weibull probability density function $f(N)$ and cumulative distribution function $F(N)$ of the fatigue life of AASC $N$ under the same cyclic loading can be expressed by eqs. (4) and (5), respectively [30, 31]:

$$
\begin{aligned}
& f(N)=\frac{\alpha}{N_{a}-N_{0}}\left[\frac{N-N_{0}}{N_{a}-N_{0}}\right]^{\alpha-1} \exp \left\{-\left[\frac{N-N_{0}}{N_{a}-N_{0}}\right]^{\alpha}\right\} \\
& F(N)=1-\exp \left\{-\left[\frac{N-N_{0}}{N_{a}-N_{0}}\right]^{\alpha}\right\}
\end{aligned}
$$

where $\alpha$ represents the Weibull shape parameter, $N_{a}$ represents the size or characteristic life parameter, and $N_{0}$ represents the position or minimum life parameter, where $N_{0}<N$.

The cumulative distribution function $F(N)$ represents the probability that the fatigue life is less than $N$, which is equivalent to the failure probability function of the sample. Thus, the reliability probability function of the sample $P(N)$ can be expressed by eq. (6). By substituting eq. (5) into eq. (6), the reliability probability function $P(N)$ with a reliability of $P$ can be obtained, as given in eq. (7).

$$
\begin{aligned}
& P(N)=1-F(N), \\
& P(N)=\exp \left\{-\left[\frac{N_{p}-N_{0}}{N_{a}-N_{0}}\right]^{\alpha}\right\},
\end{aligned}
$$

where $N_{p}$ represents the fatigue life with reliability $P$.

From the perspective of reliability, it is assumed that the minimum life of AASC due to the dispersion in AASC material strength and the variability in fatigue load tend to be 0 ; therefore, it is advisable that $N_{0}=0$. Then, the probability density function $f(N)$ and reliability probability function $\mathrm{P}(\mathrm{N})$ can be simplified into eqs. (8) and (9), respectively:

$$
\begin{aligned}
& f(N)=\frac{\alpha}{N_{a}}\left[\frac{N}{N_{a}}\right]^{\alpha-1} \exp \left\{-\left[\frac{N}{N_{a}}\right]^{\alpha}\right\}, \\
& P(N)=1-\exp \left\{-\left[\frac{N_{p}}{N_{a}}\right]^{\alpha}\right\} .
\end{aligned}
$$

Eq. (10) can be further simplified as

$$
\ln \left[\ln \left(\frac{1}{p}\right)\right]=\alpha \ln N_{p}-\alpha \ln N_{a} .
$$

Let $Y=\ln [\ln (1 / p)], X=\ln N_{p}$, and. $\beta=\alpha \ln N_{a}$ Then, eq. (10) can be written as follows:

$$
Y=\alpha X-\beta \text {. }
$$

Eq. (11) is a linear equation, and the parameters $\alpha$ and $\beta$ can be obtained by fitting the test data. Therefore, eq. (11) can be used to assess whether the test data obey the twoparameter Weibull distribution. If $Y$ and $X$ have a good linear relationship, it can be proven that the two-parameter Weibull model is suitable for this case.

In addition, the average rank should be taken as the estimation of the failure rate in the calculation, and the number $(K)$ of fatigue life test data obtained from the fatigue test under a given stress level should be arranged in increasing order with the serial number $i$. The reliability $P$ corresponding to the fatigue life $\mathrm{Np}$ can be calculated according to

$$
P=1-\frac{i}{K+1} \text {. }
$$

4.2. Two-Parameter Weibull Distribution Test. Based on the previously mentioned fatigue life test data, the relationship between the fatigue life $\mathrm{Np}$ and the corresponding reliability $p$ of AASC samples without and with frost damage under different stress levels is shown in Tables 6 and 7.

Taking $\ln [\ln (1 / P)]$ as the ordinate and $\ln N_{p}$ as the abscissa, a linear regression analysis was performed according to the data in Tables 6 and 7 to obtain the twoparameter Weibull distribution line of the flexural fatigue life of AASC, as shown in Figure 8. The correlation coefficients of $\ln [\ln (1 / P)]$ and $\ln N_{p}$ were above 0.8933 for the flexural fatigue life of all the AASC samples under different stress levels, indicating that the flexural fatigue life of AASC follows the two-parameter Weibull distribution.

The corresponding parameters of the two-parameter Weibull distribution function can be obtained using the graphical method. The estimated values of the flexural fatigue life distribution function of the undamaged and frostdamaged AASC under different stress levels are listed in Table 8.

The influences of stress level and frost damage on the shape parameters of the AASC two-parameter Weibull distribution are listed in Table 8, and there is no clear relationship. Previous studies have shown that shape parameters reflect the dispersion in the flexural fatigue life, with a higher shape parameter indicating a lower dispersion in the flexural fatigue life [32]. When the stress level was 0.70 , the shape 
TABLE 6: Two-parameter Weibull distribution test of the fatigue life of undamaged AASC.

\begin{tabular}{|c|c|c|c|c|c|}
\hline$S$ & $i$ & $N_{i}$ & $\ln N_{i}$ & $P$ & {$[\ln (1 / p)]$} \\
\hline \multirow{6}{*}{0.70} & 1 & 196088 & 12.18631 & 0.85714 & -1.86982 \\
\hline & 2 & 239314 & 12.38553 & 0.71428 & -1.08924 \\
\hline & 3 & 247521 & 12.41925 & 0.57142 & -0.58050 \\
\hline & 4 & 387625 & 12.86779 & 0.42857 & -0.16570 \\
\hline & 5 & 465893 & 13.05171 & 0.28571 & 0.22535 \\
\hline & 6 & 724824 & 13.49368 & 0.14285 & 0.66573 \\
\hline \multirow{6}{*}{0.75} & 1 & 65802 & 11.09440 & 0.85714 & -1.86982 \\
\hline & 2 & 71825 & 11.18198 & 0.71428 & -1.08924 \\
\hline & 3 & 97460 & 11.48719 & 0.57142 & -0.58050 \\
\hline & 4 & 99129 & 11.50417 & 0.42857 & -0.16570 \\
\hline & 5 & 105895 & 11.57020 & 0.28571 & 0.22535 \\
\hline & 6 & 153130 & 11.93904 & 0.14285 & 0.66573 \\
\hline \multirow{6}{*}{0.80} & 1 & 3785 & 8.23880 & 0.85714 & -1.86982 \\
\hline & 2 & 8142 & 9.00479 & 0.71428 & -1.08924 \\
\hline & 3 & 17592 & 9.77520 & 0.57142 & -0.58050 \\
\hline & 4 & 24321 & 10.09909 & 0.42857 & -0.16570 \\
\hline & 5 & 28427 & 10.25509 & 0.28571 & 0.22535 \\
\hline & 6 & 71825 & 11.18198 & 0.14285 & 0.66573 \\
\hline \multirow{6}{*}{0.85} & 1 & 649 & 6.47543 & 0.85714 & -1.86982 \\
\hline & 2 & 993 & 6.90073 & 0.71428 & -1.08924 \\
\hline & 3 & 1065 & 6.97073 & 0.57142 & -0.58050 \\
\hline & 4 & 2325 & 7.75147 & 0.42857 & -0.16570 \\
\hline & 5 & 2925 & 7.98105 & 0.28571 & 0.22535 \\
\hline & 6 & 6483 & 8.77693 & 0.14285 & 0.66573 \\
\hline
\end{tabular}

Taвle 7: Two-parameter Weibull distribution test of the fatigue life of frost-damaged AASC.

\begin{tabular}{|c|c|c|c|c|c|c|c|c|c|}
\hline \multirow{2}{*}{$S$} & \multirow{2}{*}{$i$} & \multicolumn{2}{|c|}{$n=50$} & \multicolumn{2}{|c|}{$n=100$} & \multicolumn{2}{|c|}{$n=150$} & \multirow{2}{*}{$P$} & \multirow{2}{*}{$\ln [\ln (1 / p)$} \\
\hline & & $N_{i}$ & $\ln N_{i}$ & $N_{i}$ & $\ln N_{i}$ & $N_{i}$ & $\ln N_{i}$ & & \\
\hline \multirow{6}{*}{0.70} & 1 & 90865 & 11.41713 & 61950 & 11.03408 & 26378 & 10.18028 & 0.85714 & -1.86982 \\
\hline & 2 & 164865 & 12.01288 & 112750 & 11.63292 & 58932 & 10.98414 & 0.71428 & -1.08924 \\
\hline & 3 & 255060 & 12.44925 & 184567 & 12.12576 & 85668 & 11.35823 & 0.57142 & -0.58050 \\
\hline & 4 & 290209 & 12.57835 & 233058 & 12.35904 & 103671 & 11.54897 & 0.42857 & -0.16570 \\
\hline & 5 & 354585 & 12.77870 & 261971 & 12.47598 & 113590 & 11.64035 & 0.28571 & 0.22535 \\
\hline & 6 & 584369 & 13.27828 & 475382 & 13.07187 & 260832 & 12.47163 & 0.14285 & 0.66573 \\
\hline \multirow{6}{*}{0.75} & 1 & 18621 & 9.83204 & 8526 & 9.05087 & 4986 & 8.51438 & 0.85714 & -1.86982 \\
\hline & 2 & 39435 & 10.58240 & 18690 & 9.83574 & 6750 & 8.81729 & 0.71428 & -1.08924 \\
\hline & 3 & 56806 & 10.94739 & 32795 & 10.39803 & 23954 & 10.08389 & 0.57142 & -0.58050 \\
\hline & 4 & 71796 & 11.18158 & 69816 & 11.15361 & 25685 & 10.15366 & 0.42857 & -0.16570 \\
\hline & 5 & 132698 & 11.79583 & 89830 & 11.40567 & 53581 & 10.88895 & 0.28571 & 0.22535 \\
\hline & 6 & 165615 & 12.01742 & 157327 & 11.96608 & 55376 & 10.92190 & 0.14285 & 0.66573 \\
\hline \multirow{6}{*}{0.80} & 1 & 4693 & 8.45382 & 3448 & 8.40021 & 1005 & 6.91274 & 0.85714 & -1.86982 \\
\hline & 2 & 6685 & 8.80762 & 4822 & 8.48094 & 1699 & 7.43779 & 0.71428 & -1.08924 \\
\hline & 3 & 9765 & 9.18656 & 5769 & 8.66025 & 3025 & 8.01466 & 0.57142 & -0.58050 \\
\hline & 4 & 14065 & 9.55144 & 8650 & 9.06531 & 4155 & 8.33206 & 0.42857 & -0.16570 \\
\hline & 5 & 37920 & 10.54323 & 17423 & 9.76554 & 5562 & 8.62371 & 0.28571 & 0.22535 \\
\hline & 6 & 49871 & 10.81719 & 25866 & 10.16068 & 12725 & 9.45132 & 0.14285 & 0.66573 \\
\hline
\end{tabular}

parameters decreased as the frost damage became more severe, indicating that the frost damage increased the dispersion in the flexural fatigue life of AASC at a lower stress level. However, when the stress level was 0.80 , the shape parameters were generally low $(0.8844 \sim 1.1269)$, indicating that the flexural fatigue life of AASC is more discrete under high stress levels. This may be caused by the inhomogeneity of the AASC sample; under the action of a high stress level, there was an increase in the formation rate of microcracks and a higher crack propagation rate in the sample, leading to a larger number of accumulated cracks of different degrees in the sample. This accelerated failure of the AASC and increased the dispersion in the flexural fatigue life.

4.3. Three-Parameter Weibull Distribution Theory of Flexural Fatigue Life. The two-parameter Weibull distribution can be easily obtained, but the values of the two-parameter Weibull 


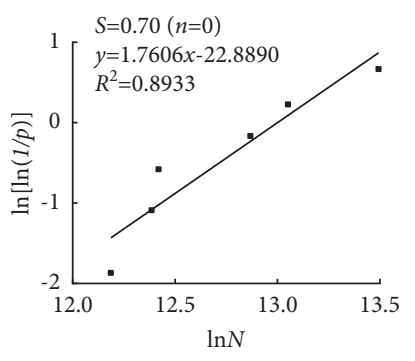

(a)

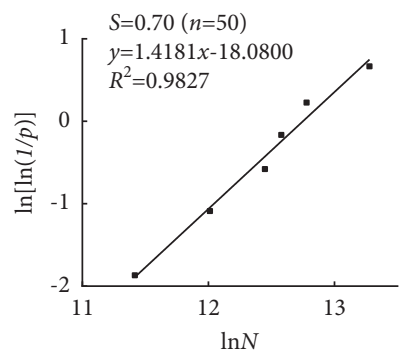

(e)

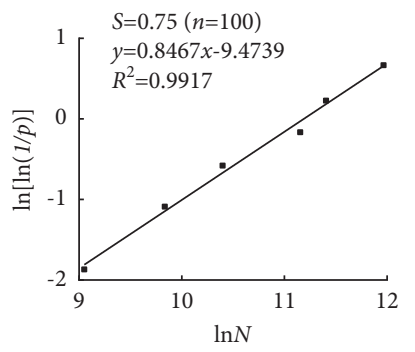

(i)

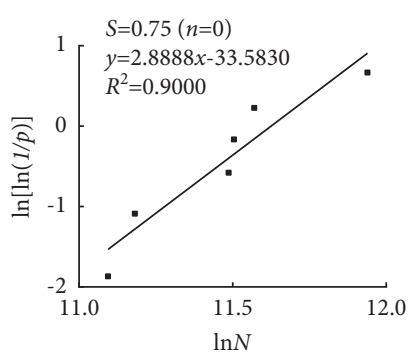

(b)

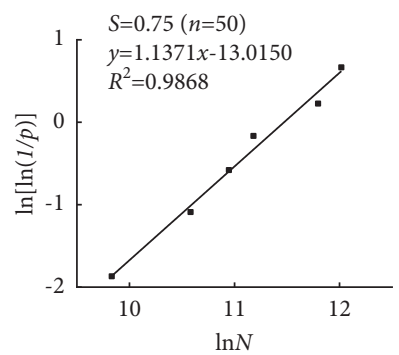

(f)

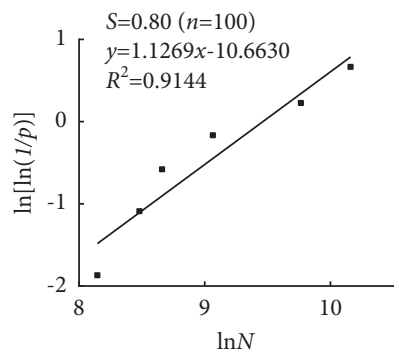

(j)

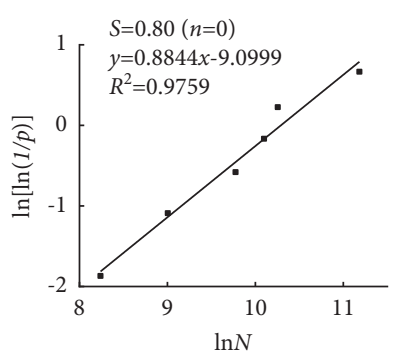

(c)

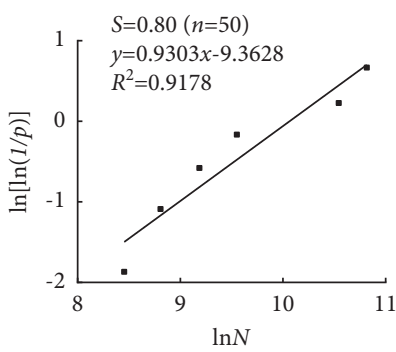

(g)

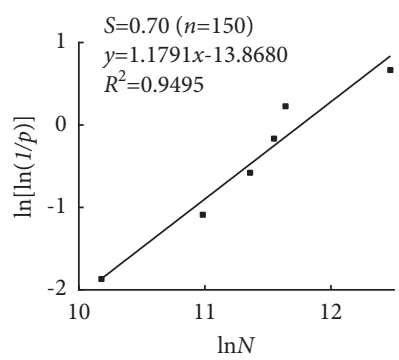

(k)

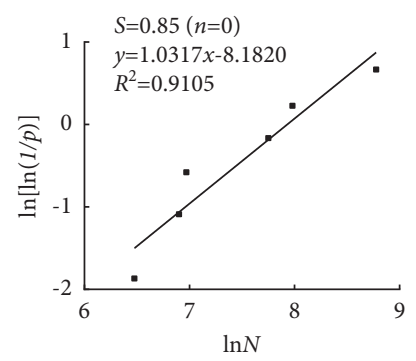

(d)

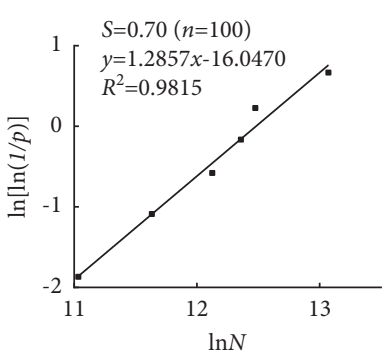

(h)

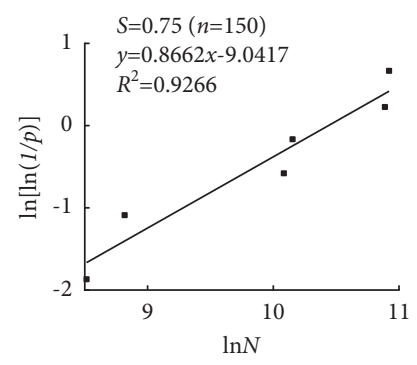

(1)

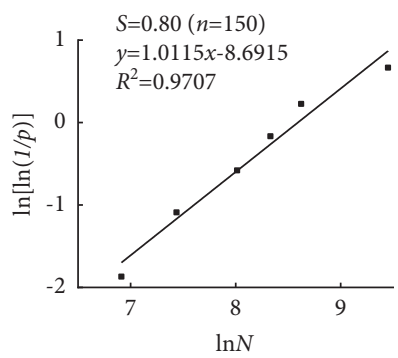

(m)

FiguRE 8: Two-parameter Weibull distribution test of the fatigue life of AASC. (a) $S=0.70(n=0), y=1.7606 x-22.8890, R 2=0.8933$; (b) $S=0.75 \quad(n=0), \quad y=2.8888 x-33.5830, \quad R 2=0.9000 ; \quad(c) \quad S=0.80 \quad(n=0), \quad y=0.8844 x-9.0999, \quad R 2=0.9759 ; \quad(d) \quad S=0.85 \quad(n=0)$, $y=1.0317 x-8.1820, \quad R 2=0.9105$; (e) $S=0.70 \quad(n=50), \quad y=1.4181 x-18.0800, R 2=0.9827$; (f) $S=0.75 \quad(n=50), \quad y=1.1371 x-13.0150$, $R 2=0.9868 ;(\mathrm{g}) S=0.80(n=50), y=0.9303 x-9.3628, R 2=0.9178 ;(\mathrm{h}) S=0.70 \quad(n=100), y=1.2857 x-16.0470, R 2=0.9815 ;(\mathrm{i}) S=0.75$ $(n=100), \quad y=0.8467 x-9.4739, \quad R 2=0.9917 ; \quad(\mathrm{j}) \quad S=0.80 \quad(n=100), \quad y=1.1269 x-10.6630, \quad R 2=0.9144 ; \quad(\mathrm{k}) \quad S=0.70 \quad(n=150)$, $y=1.1791 x-13.8680, R 2=0.9495$; (l) $S=0.75 \quad(n=150), \quad y=0.8662 x-9.0417, R 2=0.9266$; (m) $S=0.80 \quad(n=150), y=1.0115 x-8.6915$, $R 2=0.9707$

distribution estimated by the graphical method are not sufficiently accurate, which leads to certain errors. To improve the fitting accuracy of the Weibull distribution, the correlation coefficient optimization method was used to establish a three-parameter Weibull distribution. According to the definition of the three-parameter Weibull probability distribution function, the three-parameter Weibull probability density function $f(N)$ and reliability probability function $P(N)$ of fatigue life $N$ of the AASC sample under the same cyclic loading conditions can be expressed by the following equations, respectively [33]:

$$
\begin{aligned}
& f(N)=\frac{\beta}{\eta}\left(\frac{N-\gamma}{\eta}\right)^{\beta-1} \exp \left[-\left(\frac{N-\gamma}{\eta}\right)^{\beta}\right], \\
& P(N)=1-F(N)=\exp \left[-\left(\frac{N-\gamma}{\eta}\right)^{\beta}\right],
\end{aligned}
$$


TABLE 8: Estimated two-parameter Weibull distribution parameters of the flexural fatigue life of AASC.

\begin{tabular}{lcccccccc}
\hline & \multicolumn{2}{c}{$S=0.70$} & \multicolumn{2}{c}{$S=0.75$} & \multicolumn{2}{c}{$S=0.80$} & \multicolumn{2}{c}{$S=0.80$} \\
& $\alpha$ & $N_{a}$ & $\alpha$ & $N_{a}$ & $\alpha$ & $N_{a}$ & $\alpha$ & $N_{a}$ \\
\hline 0 & 1.7606 & 442754 & 2.8888 & 111895 & 0.8844 & 29420 & 1.0317 \\
50 & 1.4181 & 344393 & 1.1371 & 93513 & 0.9303 & 23490 & - \\
100 & 1.2857 & 263345 & 0.8467 & 72351 & 1.1269 & 12866 & - \\
150 & 1.1791 & 128231 & 0.8662 & 34147 & 1.0115 & 5392 & - \\
\hline
\end{tabular}

where $\gamma$ represents the position parameter, $\eta$ represents the scale parameter, and $\beta$ represents the shape parameter. Eq. (14) is further simplified to obtain

$$
\ln \left[\ln \left(\frac{1}{P}\right)\right]=\beta \ln (N-\gamma)-\beta \ln \eta .
$$

Let $Y=\ln [\ln (1 / P)], \quad X=\ln (N-\gamma), a=-\beta \ln \eta$, and $b=\beta$. Then, eq. (15) can be written as

$$
Y=a+b X \text {. }
$$

The reliability corresponding to fatigue life $N_{p}$ was calculated according to eq. (12). The distribution parameters were calculated using the correlation coefficient optimization method. According to eq. (16), it can be seen that there is a linear relationship between the variables $X$ and $Y$.The corresponding correlation coefficients $R(x, y)$ of $X$ and $Y$ are shown in

$$
R(x, y)=\frac{\sum_{i=1}^{n} x_{i} y_{i}-n \bar{x} \bar{y}}{\sqrt{\left(\sum_{i=1}^{n} x_{i}^{2}-n \bar{x}^{2}\right)\left(\sum_{i=1}^{n} y_{i}^{2}-n \bar{y}^{2}\right)}},
$$

where $\bar{x}=(1 / n) \sum_{i=1}^{n} x_{i}$ and $\bar{y}=(1 / n) \sum_{i=1}^{n} y_{i}$. The function with correlation coefficient $R(x, y)$ is $\gamma$, and the best linearity in $\gamma$ is obtained on the $X$ and $Y$ lines, that is, the maximum $\gamma$. Then, the least square regression can be used to find $a, b$ $(\beta)$, and $\eta$.

4.4. Three-Parameter Weibull Distribution Test. The threeparameter Weibull distribution often has more physical significance to describe the probability distribution of the structural fatigue life. In structural life evaluation characterized by loss, the three-parameter Weibull distribution is more accurate than the two-parameter Weibull distribution. Therefore, to reflect the probability distribution of the flexural fatigue life of AASC more comprehensively and accurately, a three-parameter Weibull distribution test was adopted in this study. According to the principles described in Section 4.3, MATLAB was used to solve for the parameters of the Weibull distribution, which is more convenient and accurate.

The total curve of the Weibull distribution $\ln [\ln (1 / P)]$ and $\ln (N-\gamma)$ for the three parameters of the flexural fatigue life of AASC is shown in Figure 9. Table 9 shows the regression coefficients and correlation coefficients of the fatigue life of AASC with various degrees of frost damage at various stress levels obtained by regression analysis of the three-parameter Weibull distribution test data.

$\beta$ is the shape parameter, and a smaller $\beta$ represents a greater dispersion in the distribution function [34]. As shown in Table 9, the shape parameters of the AASC samples subjected to frost damage decreased as the stress level increased, indicating that the dispersion in the flexural fatigue life increased. More serious frost damage resulted in smaller shape parameters, which indicates that with the aggravation of frost damage, and the dispersion in the flexural fatigue life of AASC gradually increases. This may be due to random failures inside the sample after frost damage of the AASC. Factors related to frost damage from freeze-thaw cycles, such as hydrostatic pressure and seepage pressure, could lead to random degradation of the interface transition area because the sample itself is nonhomogeneous. There is a certain randomness in frost damage; thus, the internal structure and properties of AASC become more uneven after frost damage, which explains why more serious frost damage yielded a greater discreteness in the flexural fatigue life.

In addition, $\eta$ represents the scale parameter, which magnifies or shrinks the curve but does not affect its shape. A larger value of $\eta$ yields a greater dispersion in the distribution function. $\eta$ has a strong regularity in Table 9; that is, with increasing stress level, and $\eta$ decreases, and the discreteness of the flexural fatigue life data decreases.

By comparing the Weibull distribution models in Sections 4.2 and 4.4, it was found that the fatigue life of AASC follows both the two-parameter and three-parameter Weibull distributions, but the linear fitting degree and correlation coefficient of the three-parameter Weibull distribution are better than those of the two-parameter Weibull distribution. This shows that the three-parameter Weibull distribution method can reflect the probability distribution of the fatigue life of AASC more accurately.

4.5. Fatigue Life Distribution Fitting Test. The flexural fatigue life of AASC follows both the two-parameter and threeparameter Weibull distributions, but the fitting test method should be used to further verify whether the flexural fatigue life distribution function calculated by the above method could meet the calculation requirements. Because the flexural fatigue life is a one-dimensional random variable and the theoretical distribution of a small sample size is a completely known continuous distribution, the $K-S$ test method is appropriate [35]. The fit distribution of the flexural fatigue life of AASC was compared with the measured distribution to determine whether the distribution parameters and sample distribution determined by the graphical method and correlation coefficient method were consistent with the Weibull distribution.

The $K-S$ test method was used to further assess the distribution of the flexural fatigue life of AASC. It was 


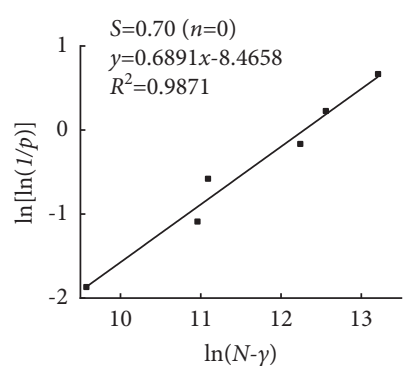

(a)

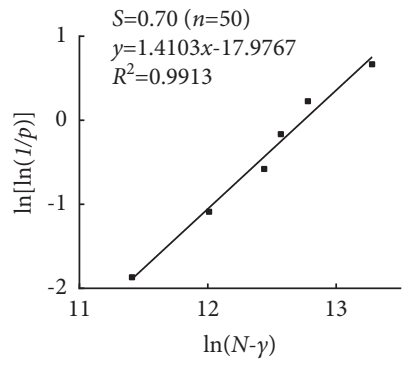

(e)

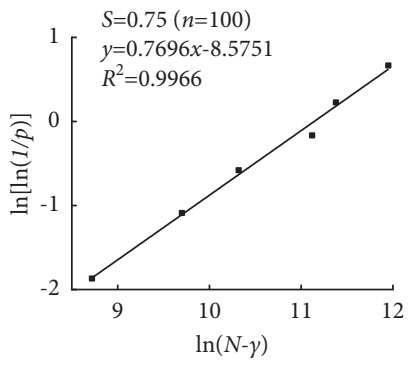

(i)

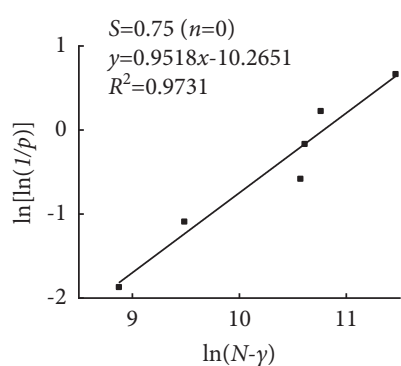

(b)

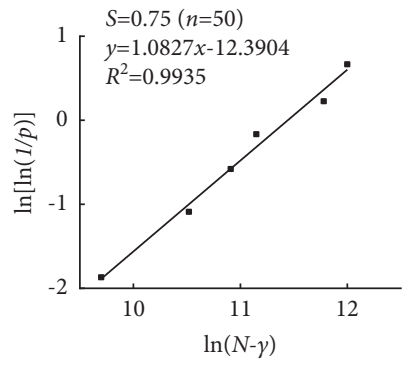

(f)

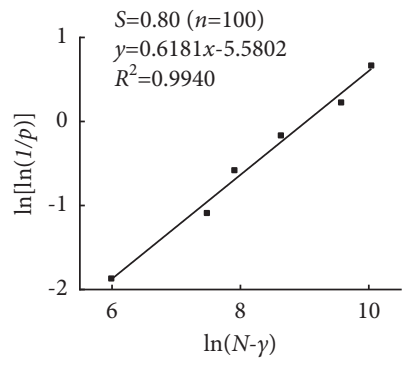

(j)

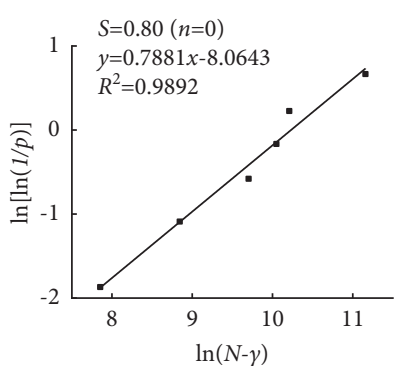

(c)

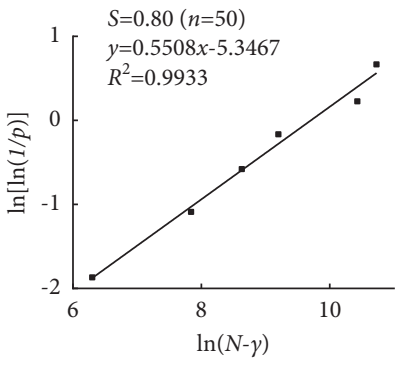

(g)

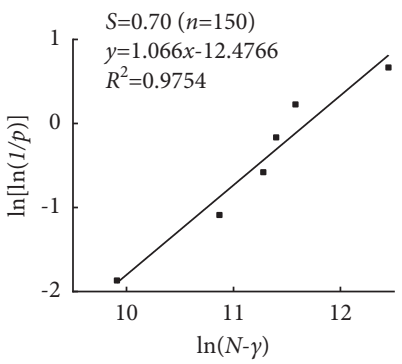

(k)

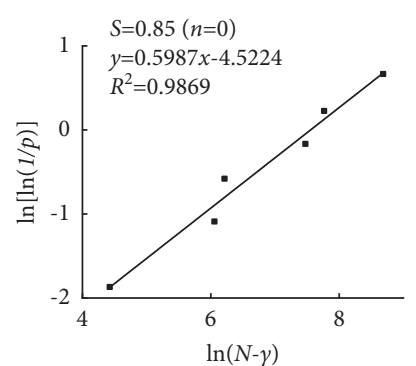

(d)

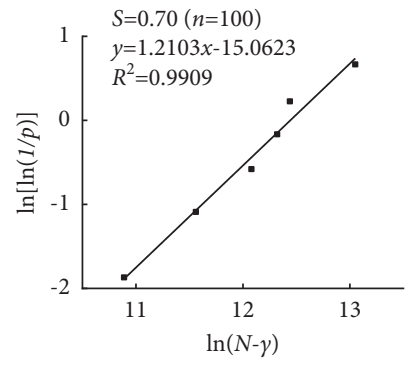

(h)

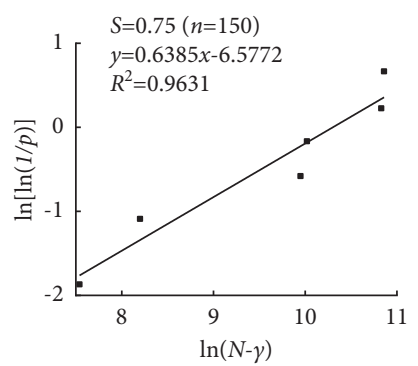

(l)

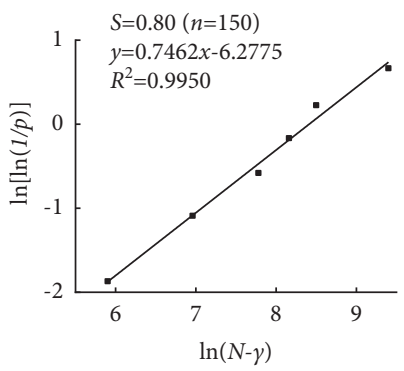

(m)

Figure 9: Three-parameter Weibull distribution test of the fatigue life of AASC. (a) $S=0.70(n=0), y=0.6891 x-8.4658, R 2=0.9871$; (b) $S=0.75(n=0), \quad y=0.9518 x-10.2651, \quad R 2=0.9731 ; \quad(c) S=0.80 \quad(n=0), \quad y=0.7881 x-8.0643, R \quad 2=0.9892 ; \quad(d) \quad S=0.85 \quad(n=0)$, $y=0.5987 x-4.5224, R 2=0.9869$; (e) $S=0.70 \quad(n=50), y=1.4103 x-17.9767, R 2=0.9913$; (f) $S=0.75 \quad(n=50), y=1.0827 x-12.3904, R$ $2=0.9935 ;(\mathrm{g}) S=0.80(n=50), y=0.5508 x-5.3467, R 2=0.9933$; (h) $S=0.70(n=100), y=1.2103 x-15.0623, R 2=0.9909$; (i) $S=0.75$ $(n=100), y=0.7696 x-8.5751, R 2=0.9966$; (j) $S=0.80(n=100), y=0.6181 x-5.5802, R 2=0.9940$; (k) $S=0.70(n=150), y=1.066 x-12.4766$, $R 2=0.9754$; (l) $S=0.75$ ( $n=150), y=0.6385 x-6.5772, R 2=0.9631$; (m) $S=0.80(n=150), y=0.7462 x-6.2775, R 2=0.9950$.

assumed that $F\left(N_{i}\right)$ and $F_{k}\left(N_{i}\right)$ were cumulative probability distribution functions of the total and theoretical distributions, respectively. $D_{i}$ represents the maximum difference between the cumulative probability distribution function of the population and the theoretical distribution. The maximum deviation $D_{i}$ as a statistic is shown in

$$
D_{i}=\max _{i}^{k}\left\{\left|F\left(N_{i}\right)-F_{k}\left(N_{i}\right)\right|\right\} .
$$

For a given significance level $\alpha, D_{i}$ is a fixed constant $D_{i, \alpha}$. When $D_{i}>D_{i, \alpha}$, the test is negative and vice versa. The $K-S$ test method was used to assess the fitting of the flexural fatigue life distribution of AASC. By examining the sample $K-S$ test statistical scale, it was found that $D_{i, 0.05}=0.519$ when the sample size was six and the significance level was 0.05 . The results of the two-parameter and three-parameter Weibull distribution tests of the flexural fatigue life of AASC are presented in Table 10. 
TABle 9: Three-parameter Weibull distribution test analytic results.

\begin{tabular}{|c|c|c|c|c|c|}
\hline$n$ & Regression coefficient & $S=0.70$ & $S=0.75$ & $S=0.80$ & $S=0.85$ \\
\hline \multirow{4}{*}{0} & $\gamma$ & 181691.3367 & 58655.1510 & 1211.6878 & 565.4457 \\
\hline & $\beta$ & 0.6891 & 0.9518 & 0.7881 & 0.5897 \\
\hline & $\eta$ & 216533.9464 & 48296.2997 & 27795.6383 & 1907.9167 \\
\hline & $R^{2}$ & 0.9871 & 0.9731 & 0.9892 & 0.9869 \\
\hline \multirow{4}{*}{50} & $\gamma$ & 1086.6727 & 2260.6583 & 4149.1147 & - \\
\hline & $\beta$ & 1.4103 & 1.0827 & 0.5508 & - \\
\hline & $\eta$ & 343315.2946 & 93338.5199 & 16423.8744 & - \\
\hline & $R^{2}$ & 0.9913 & 0.9935 & 0.9933 & - \\
\hline \multirow{4}{*}{100} & $\gamma$ & 8354.1341 & 2374.5490 & 3050.0740 & - \\
\hline & $\beta$ & 1.2103 & 0.7696 & 0.6181 & - \\
\hline & $\eta$ & 253980.3397 & 69016.5545 & 8329.9645 & - \\
\hline & $R^{2}$ & 0.9909 & 0.9966 & 0.9940 & - \\
\hline \multirow{4}{*}{150} & $\gamma$ & 6205.3262 & 3105.3380 & 641.2403 & - \\
\hline & $\beta$ & 1.0660 & 0.6385 & 0.7462 & - \\
\hline & $\eta$ & 121115.9899 & 29789.9436 & 4501.7225 & - \\
\hline & $R^{2}$ & 0.9754 & 0.9631 & 0.9950 & - \\
\hline
\end{tabular}

TABLE 10: Two-parameter and three-parameter Weibull distributions tests of the fatigue life of AASC.

\begin{tabular}{cccc}
\hline$n$ & $S$ & Two-parameter weibull $D_{i}$ & Three-parameter weibull $D_{i}$ \\
\hline \multirow{4}{*}{0} & 0.70 & 0.1982 & 0.1519 \\
& 0.75 & 0.2595 & 0.2090 \\
& 0.80 & 0.2124 & 0.2073 \\
50 & 0.85 & 0.1897 & 0.1552 \\
& 0.70 & 0.1860 & 0.1860 \\
& 0.75 & 0.1473 & 0.1599 \\
100 & 0.80 & 0.2043 & 0.1725 \\
& 0.70 & 0.2037 & 0.2018 \\
& 0.75 & 0.1451 & 0.1551 \\
& 0.80 & 0.1943 & 0.1550 \\
& 0.70 & 0.2536 & 0.2486 \\
& 0.75 & 0.2187 & 0.2389 \\
& 0.80 & 0.1897 & 0.1768 \\
\hline
\end{tabular}

According to the test results in Table 10, the two-parameter and three-parameter Weibull distribution test results of the flexural fatigue life of AASC under various degrees of frost damage at various stress levels all follow the Weibull distribution. In addition, the three-parameter Weibull test value $D_{i}$ was smaller in terms of the test effect; therefore, the three-parameter Weibull distribution is more effective.

\section{Reliability Analysis of Flexural Fatigue}

The reliability probability of the previously mentioned flexural fatigue life equation, the AASC average flexural fatigue life equation, is close to $50 \%$. This cannot meet the requirements of structural fatigue life verification and design. Therefore, according to the previously mentioned flexural fatigue life equation, fatigue life equations with different failure probabilities should be calculated and designed to meet the requirements of fatigue design and other related requirements.
5.1. $\mathbf{P}-\lg \mathbf{S}-\lg \mathbf{N}$ and $\mathbf{P}-\lg \mathbf{S}-\lg \mathbf{N}$ Curves of the Two-Parameter Weibull Distribution. According to the results presented in Section 3, the flexural fatigue life of AASC $\left(N_{p}\right)$ obeys a two-parameter Weibull distribution, and eq. (9) can be used to obtain the corresponding equivalent fatigue life. According to the different degrees of freeze-thaw damage to AASC, eq. (19) can be obtained for a given failure probability $P_{f}$ and flexural fatigue life $N_{p}$. Table 11 shows the effects of failure probability on the flexural fatigue life of AASC [28]:

$$
N_{p}=N_{a}\left[\ln \left(\frac{1}{P}\right)\right]^{1 / b} .
$$

Based on the calculated equivalent fatigue lives, the flexural fatigue equations $S \lg N_{p}$ and $\lg S \lg N_{p}$ corresponding to two-parameter Weibull distributions with different failure probabilities under different stress levels of undamaged and frost-damaged AASC were obtained by regression analysis, as shown in Figures 10 and 11 and Table 12 . 
TABLE 11: Flexural fatigue life $\left(N_{p}\right)$ of AASC for various failure probabilities.

\begin{tabular}{|c|c|c|c|c|}
\hline$n$ & $P_{f}$ & $S=0.70$ & $S=0.75$ & $S=0.80$ \\
\hline \multirow{6}{*}{0} & 0.05 & 81939 & 40020 & 1024 \\
\hline & 0.10 & 123326 & 51345 & 2310 \\
\hline & 0.20 & 188871 & 66576 & 5397 \\
\hline & 0.30 & 246523 & 78311 & 9171 \\
\hline & 0.40 & 302318 & 88680 & 13767 \\
\hline & 0.50 & 359545 & 9856 & 19440 \\
\hline \multirow{6}{*}{50} & 0.05 & 42406 & 6862 & 965 \\
\hline & 0.10 & 70449 & 129243 & 2091 \\
\hline & 0.20 & 119590 & 25003 & 4683 \\
\hline & 0.30 & 166468 & 37768 & 7756 \\
\hline & 0.40 & 214456 & 51799 & 11411 \\
\hline & 0.50 & 265956 & 67747 & 15841 \\
\hline \multirow{6}{*}{100} & 0.05 & 26135 & 2167 & 922 \\
\hline & 0.10 & 45749 & 5072 & 1746 \\
\hline & 0.20 & 82009 & 1205 & 3399 \\
\hline & 0.30 & 118111 & 21412 & 5154 \\
\hline & 0.40 & 156180 & 32726 & 7088 \\
\hline & 0.50 & 198026 & 46930 & 9293 \\
\hline \multirow{6}{*}{150} & 0.05 & 10327 & 1107 & 286 \\
\hline & 0.10 & 19016 & 2541 & 583 \\
\hline & 0.20 & 35936 & 6044 & 1224 \\
\hline & 0.30 & 53490 & 10386 & 1946 \\
\hline & 0.40 & 72540 & 15724 & 2776 \\
\hline & 0.50 & 93972 & 22366 & 3753 \\
\hline
\end{tabular}

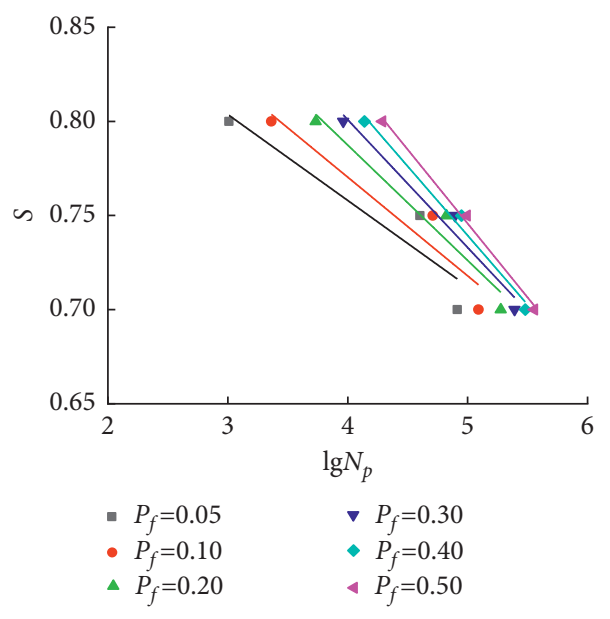

(a)

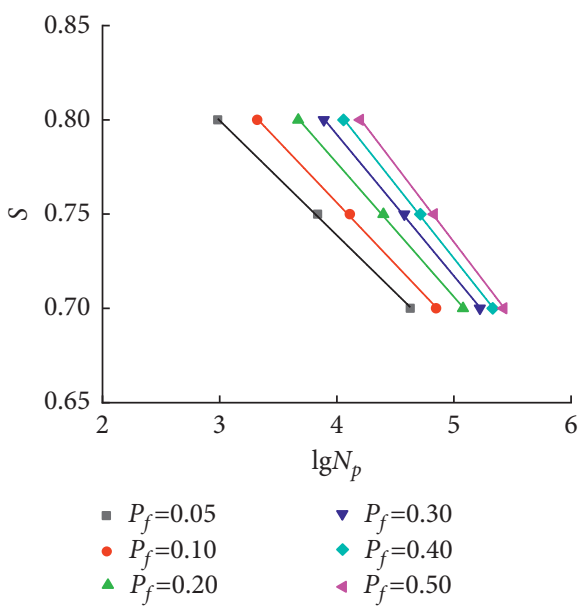

(b)

Figure 10: Continued. 


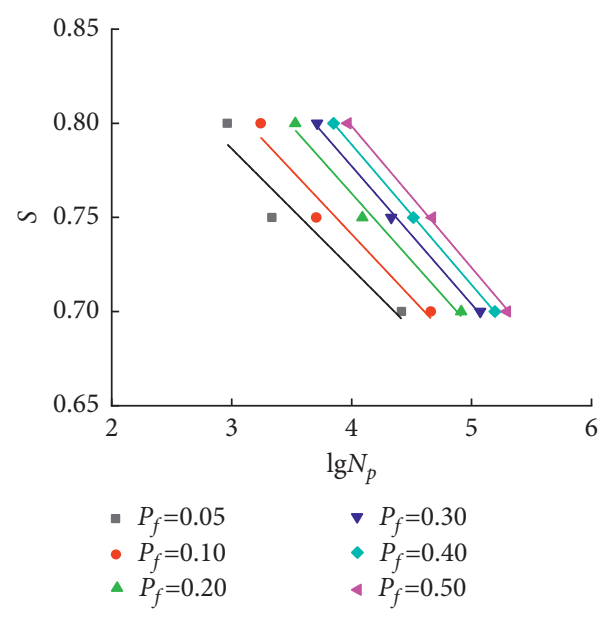

(c)

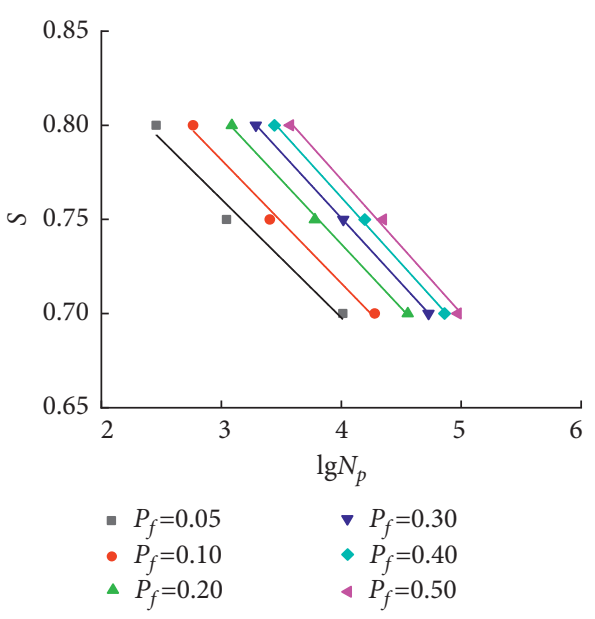

(d)

FIgURE 10: $S-\operatorname{lgN}$ pelationships of AASC with various degrees of frost damage for various failure probabilities. (a) $n=0$. (b) $n=50$. (c) $n=100$. (d) $n=150$.

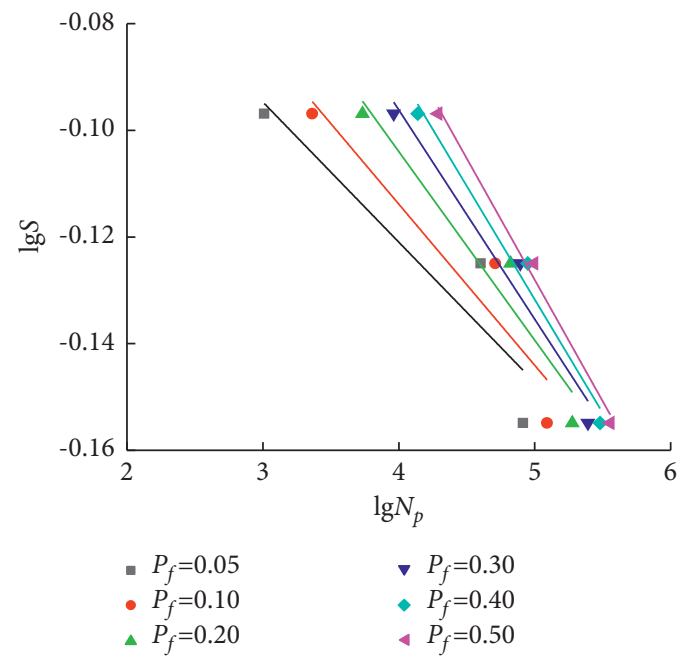

(a)

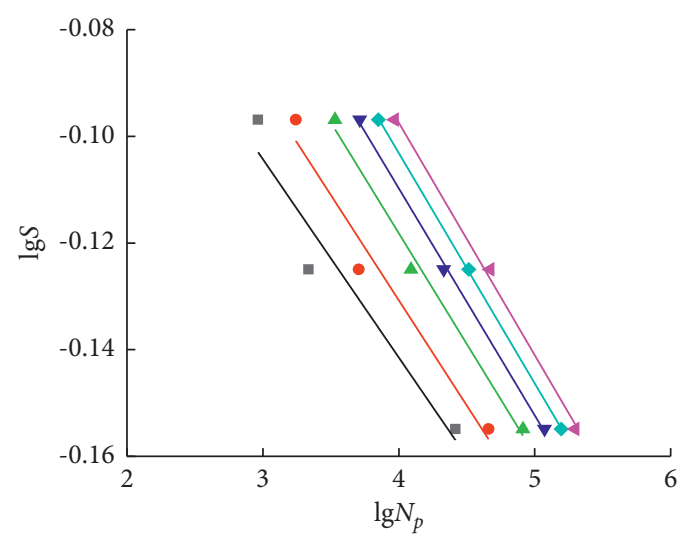

$$
\begin{aligned}
\text { - } P_{f}=0.05 \\
\text { - } P_{f}=0.10 \\
\text { ॥ } P_{f}=0.20
\end{aligned}
$$

(c)

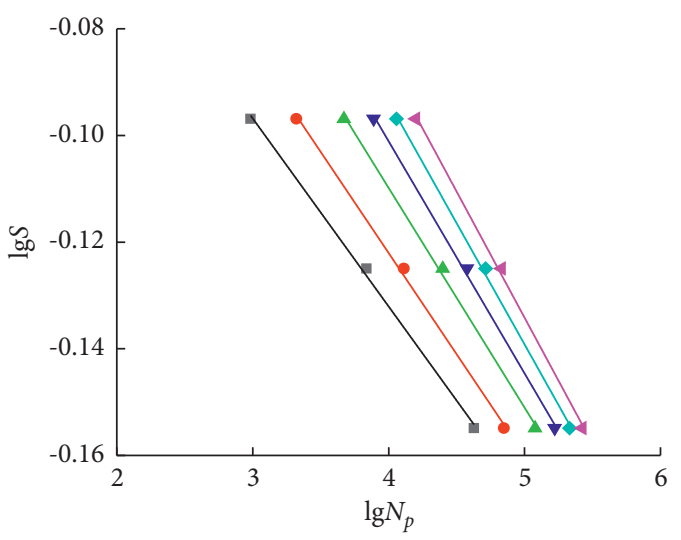
- $P_{f}=0.05$
- $P_{f}=0.10$
> $P_{f}=0.30$
$\triangle P_{f}=0.20$
- $P_{f}=0.40$
\ $P_{f}=0.50$

(b)

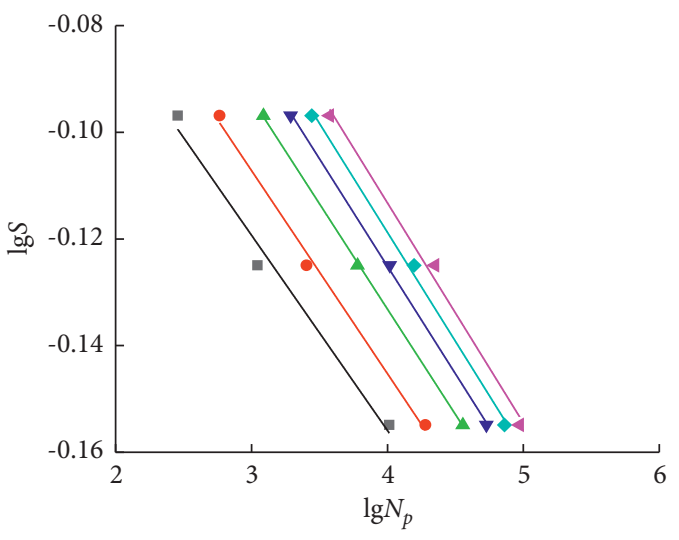

$$
\begin{array}{ll}
\text { - } P_{f}=0.05 & \text { จ } P_{f}=0.30 \\
\text { - } P_{f}=0.10 & \diamond P_{f}=0.40 \\
\Delta P_{f}=0.20 & \text { \& } P_{f}=0.50
\end{array}
$$

(d)

FIGURE 11: $\operatorname{lgS}-\lg \mathrm{N}_{\mathrm{p}}$ relationships of AASC with various degrees of frost damage for various failure probabilities. (a) $n=0$. (b) $n=50$. (c) $n=100$. (d) $n=150$. 
TABLE 12: Single-logarithmic and double-logarithmic fatigue equations of AASC with various degrees of frost damage for various failure probabilities.

\begin{tabular}{|c|c|c|c|c|c|}
\hline$n$ & $P_{f}$ & Single-logarithmic fatigue equation & $R^{2}$ & Double-logarithmic fatigue equation & $R^{2}$ \\
\hline \multirow{6}{*}{0} & 0.05 & $S=0.9406-0.0456 \lg N$ & 0.8688 & $\lg S=0.0159-0.0263 \lg N$ & 0.8556 \\
\hline & 0.10 & $S=0.9800-0.0524 \lg N$ & 0.9055 & $\lg S=0.0070-0.0302 \lg N$ & 0.8940 \\
\hline & 0.20 & $S=1.0325-0.0613 \lg N$ & 0.9461 & $\lg S=0.0375-0.0354 \lg N$ & 0.9371 \\
\hline & 0.30 & $S=1.0724-0.0679 \lg N$ & 0.9703 & $\lg S=0.0607-0.0392 \lg N$ & 0.9634 \\
\hline & 0.40 & $S=1.1069-0.0735 \lg N$ & 0.9861 & $\lg S=0.0809-0.0425 \lg N$ & 0.9812 \\
\hline & 0.50 & $S=1.1387-0.0786 \lg N$ & 0.9958 & $\lg S=0.0996-0.0455 \lg N$ & 0.9929 \\
\hline \multirow{6}{*}{50} & 0.05 & $S=0.9821-0.0608 \lg N$ & 0.9995 & $\lg S=0.0090-0.0353 \lg N$ & 0.9983 \\
\hline & 0.10 & $S=1.0178-0.0654 \lg N$ & 0.9996 & $\lg S=0.0297-0.0379 \lg N$ & 0.9984 \\
\hline & 0.20 & $S=1.0613-0.0710 \lg N$ & 0.9996 & $\lg S=0.0549-0.0412 \lg N$ & 0.9985 \\
\hline & 0.30 & $S=1.0925-0.0751 \lg N$ & 0.9996 & $\lg S=0.0730-0.0435 \lg N$ & 0.9986 \\
\hline & 0.40 & $S=1.1189-0.0785 \lg N$ & 0.9997 & $\lg S=0.0883-0.0455 \lg N$ & 0.9986 \\
\hline & 0.50 & $S=1.1432-0.0816 \lg N$ & 0.9997 & $\lg S=0.1024-0.0473 \lg N$ & 0.9986 \\
\hline \multirow{6}{*}{100} & 0.05 & $S=0.9778-0.0638 \lg N$ & 0.9262 & $\lg S=0.0073-0.0372 \lg N$ & 0.9360 \\
\hline & 0.10 & $S=1.0123-0.0678 \lg N$ & 0.9614 & $\lg S=0.0271-0.0395 \lg N$ & 0.9685 \\
\hline & 0.20 & $S=1.0486-0.0715 \lg N$ & 0.9879 & $\lg S=0.0479-0.0415 \lg N$ & 0.9917 \\
\hline & 0.30 & $S=1.0705-0.0733 \lg N$ & 0.9973 & $\lg S=0.0605-0.0426 \lg N$ & 0.9989 \\
\hline & 0.40 & $S=1.0865-0.0745 \lg N$ & 0.9999 & $\lg S=0.0696-0.0432 \lg N$ & 0.9998 \\
\hline & 0.50 & $S=1.0993-0.0752 \lg N$ & 0.9989 & $\lg S=0.0768-0.0436 \lg N$ & 0.9972 \\
\hline \multirow{6}{*}{150} & 0.05 & $S=0.9496-0.0629 \lg N$ & 0.9803 & $\lg S=0.0095-0.0366 \lg N$ & 0.9853 \\
\hline & 0.10 & $S=0.9783-0.0655 \lg N$ & 0.9921 & $\lg S=0.0070-0.0381 \lg N$ & 0.9951 \\
\hline & 0.20 & $S=1.0092-0.0681 \lg N$ & 0.9990 & $\lg S=0.0248-0.0395 \lg N$ & 0.9998 \\
\hline & 0.30 & $S=1.0287-0.0695 \lg N$ & 0.9999 & $\lg S=0.0360-0.0403 \lg N$ & 0.9994 \\
\hline & 0.40 & $S=1.0436-0.0705 \lg N$ & 0.9987 & $\lg S=0.0446-0.0408 \lg N$ & 0.9969 \\
\hline & 0.50 & $S=1.0562-0.0712 \lg N$ & 0.9961 & $\lg S=0.0518-0.0413 \lg N$ & 0.9933 \\
\hline
\end{tabular}

The analysis results show that the $S \lg N_{p}$ and $\lg S \lg N_{p}$ equations of the undamaged and frost-damaged AASC under various stress levels for a given failure probability have correlation coefficients above 0.85 . This shows that the equivalent flexural fatigue life of AASC using the singlelogarithmic and double-logarithmic fatigue equations is able to follow the two-parameter Weibull distribution.

It can be observed from Figures 10 and 11 that all samples in the curves of the single-logarithmic and doublelogarithmic flexural fatigue equations show a similar rule: the flexural fatigue life decreases with an increase in the stress level and probability level at each failure probability and is in consistence with the research results of Meng Chen et al. [34]. The frost damage also has a clear effect on the flexural fatigue performance of AASC: the flexural fatigue life of AASC decreases with the aggravation of frost damage under each failure probability. As mentioned above, frost damage leads to different degrees of internal damage in AASC samples, leading to deterioration of the AASC flexural fatigue performance. Therefore, the flexural fatigue life decreases with the aggravation of freeze-thaw damage. It is worth mentioning that the flexural fatigue life of undamaged AASC at low stress levels has low discreteness, whereas the dispersion in the flexural fatigue life of frost-damaged AASC at low stress levels is higher. This is due to the mechanical performance degradation of the gelled material in frost-damaged AASC, and the frost heave pressure in the frost-damaged AASC led to the damage to the sample structure. Therefore, frost damage has a significant impact on the flexural fatigue properties of AASC. The frost damage intensifies the flexural fatigue damage in the samples and becomes more serious with an increase in the degree of frost damage.

5.2. $\mathbf{P}-\mathbf{S}-\lg \mathbf{N}$ and $\mathbf{P}-\lg \mathbf{S}-\lg \mathbf{N}$ Curves of the Three-Parameter Weibull Distribution. According to the results presented in Section 3, the flexural fatigue life of AASC $\left(N_{p}\right)$ also follows the three-parameter Weibull distribution. Therefore, its corresponding equivalent fatigue life $\left(N_{p}\right)$ can be obtained from eq. (14), as shown in eq. (20). According to eq. (20), the flexural fatigue life $N_{p}$ of AASC with various degrees of frost damage at a given failure probability can be obtained, as shown in Table 13.

$$
N_{p}=\eta\left(\ln \frac{1}{P}\right)^{1 / \beta}+\gamma
$$

Based on the calculated equivalent fatigue lives, regression analysis was performed to obtain the single-logarithmic and double-logarithmic flexural fatigue equations of the two-parameter Weibull distribution corresponding to various failure probabilities under different stress levels of undamaged and frost-damaged AASC, as shown in Figures 12 and 13 and Table 14.

The $S-\lg \mathrm{N}_{\mathrm{p}}$ and $\lg S-\lg \mathrm{N}_{\mathrm{p}}$ equations of AASC with various degrees of frost damage under various stress levels for a given failure probability of the three-parameter Weibull distribution had correlation coefficients of greater than 0.81 . This shows that the linear relationship between the singlelogarithmic and double-logarithmic flexural fatigue equations of the equivalent flexural fatigue life of AASC under the three-parameter Weibull distribution is established, and it 
TABLE 13: Flexural fatigue life $\left(N_{p}\right)$ of AASC for various failure probabilities.

\begin{tabular}{|c|c|c|c|c|}
\hline$n$ & $P_{f}$ & $S=0.70$ & $S=0.75$ & $S=0.80$ \\
\hline \multirow{6}{*}{0} & 0.05 & 184599 & 60786 & 1853 \\
\hline & 0.10 & 189957 & 63196 & 2811 \\
\hline & 0.20 & 206250 & 68644 & 5356 \\
\hline & 0.30 & 230198 & 75005 & 5726 \\
\hline & 0.40 & 263387 & 82501 & 13064 \\
\hline & 0.50 & 308906 & 91516 & 18670 \\
\hline \multirow{6}{*}{50} & 0.05 & 42873 & 8268 & 4224 \\
\hline & 0.10 & 70702 & 13939 & 4425 \\
\hline & 0.20 & 119607 & 25617 & 5228 \\
\hline & 0.30 & 166368 & 38280 & 6676 \\
\hline & 0.40 & 214312 & 52451 & 9000 \\
\hline & 0.50 & 265831 & 68795 & 12592 \\
\hline \multirow{6}{*}{100} & 0.05 & 30181 & 3829 & 3118 \\
\hline & 0.10 & 47918 & 6082 & 3269 \\
\hline & 0.20 & 81903 & 12204 & 3786 \\
\hline & 0.30 & 116714 & 20454 & 4621 \\
\hline & 0.40 & 154156 & 31208 & 5860 \\
\hline & 0.50 & 195976 & 45242 & 7654 \\
\hline \multirow{6}{*}{150} & 0.05 & 26442 & 3390 & 725 \\
\hline & 0.10 & 37427 & 3983 & 862 \\
\hline & 0.20 & 55272 & 5949 & 1245 \\
\hline & 0.30 & 71291 & 9033 & 1774 \\
\hline & 0.40 & 87015 & 13509 & 2474 \\
\hline & 0.50 & 103326 & 19885 & 3400 \\
\hline
\end{tabular}

can better follow the three-parameter Weibull distribution. In addition, it should be noted that in flexural fatigue distribution fitting (Section 3), the three-parameter Weibull distribution showed higher accuracy than the two-parameter Weibull distribution. However, by comparing the correlation coefficients obtained from the regression of the singlelogarithmic and double-logarithmic flexural fatigue equations, it was found that the three-parameter Weibull distribution did not show similar advantages in the reliability analysis, and the correlation was inferior to that of the twoparameter Weibull distribution in individual cases.

According to Figures 12 and 13, the flexural fatigue equation relations $S-\lg \mathrm{N}_{\mathrm{p}}$ and $\lg S-\lg \mathrm{N}_{\mathrm{p}}$ of the three-parameter Weibull distribution are essentially similar to those of the two-parameter Weibull distribution. The flexural fatigue life decreases with an increase in the stress level and probability level when the failure probability is constant. In addition, frost damage has a significant impact on the AASC flexural fatigue properties and intensifies the flexural fatigue damage of the samples. The damage becomes more serious with increasing degree of frost damage.

By consulting references, it was found that any standard does not explicitly give the failure probability of flexural fatigue life that should be considered in the design. To ensure reliable flexural fatigue design strength, $P_{f}=0.05$ is typically considered as the ultimate flexural fatigue strength [36], and Goel and Singh [37] reported that the lowest failure probability should be chosen to the fatigue design. Therefore, the
$S-\lg \mathrm{N}_{\mathrm{p}}$ and $\lg S-\lg \mathrm{N}_{\mathrm{p}}$ curves for various degrees of frost damage at $P_{f}=0.05$ are plotted in Figure 14 .

As shown in Figure 14, when $P_{f}=0.05$, the flexural fatigue life of AASC decreased with increasing stress level. The dispersion in the flexural fatigue life is lower at higher stress levels but becomes larger at lower stress levels. When the stress level was 0.7, the flexural fatigue lives of the AASC samples that underwent 50,100, and 150 freeze-thaw cycles in the $\lg S-\lg \mathrm{N}_{\mathrm{p}}$ diagram were reduced by $13.9 \%, 36.6 \%$, and $66.6 \%$, respectively, compared with those of the samples that did not experience freeze-thaw cycles. When the stress level was 0.7 , comparison of the $\lg S-\lg \mathrm{N}_{\mathrm{p}}$ curves of the undamaged samples and the frost-damaged samples after at 50 , 100 , and 150 freeze-thaw cycles indicates that the flexural fatigue life was reduced by $13.9 \%, 36.6 \%$, and $66.6 \%$, respectively. It is worth noting that when $P_{f}=0.05$ was at the high stress level, the flexural fatigue life of the undamaged sample was lower than those of the samples that underwent 50 and 100 freeze-thaw cycles. However, when $P_{f}>0.30$, the flexural fatigue life of the undamaged sample was higher than that of the frost-damaged sample. This shows that when $P_{f}$ is small, the flexural fatigue life of AASC is more sensitive to frost damage at high stress. Combined with the experimental results in Section 3, it is concluded that the sample is more prone to form microcracks and has a higher crack growth rate under higher stress levels, which leads to the acceleration of the flexural fatigue failure to varying degrees, thus increasing the dispersion in the flexural fatigue life. 

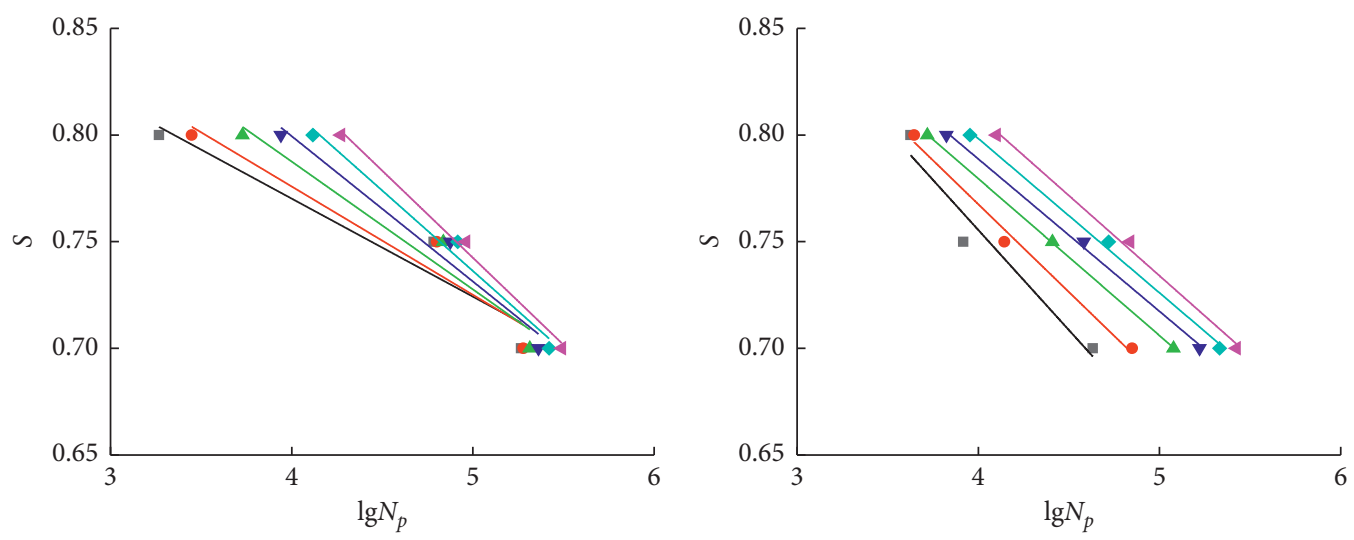

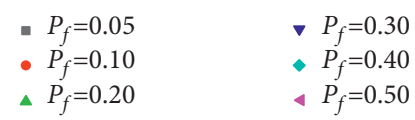

(a)

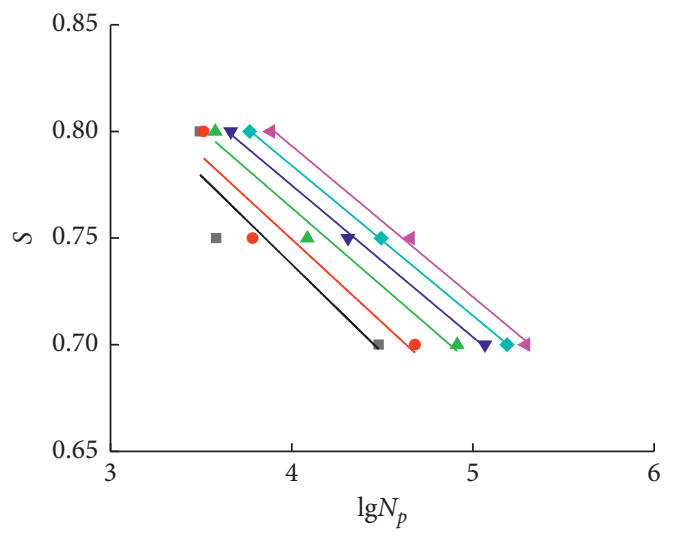

- $P_{f}=0.05$

- $P_{f}=0.10$

^ $P_{f}=0.20$

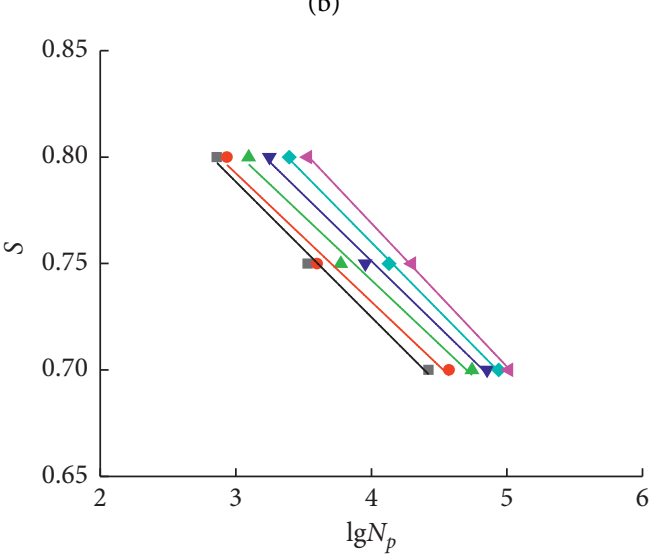

- $P_{f}=0.05$

- $P_{f}=0.10$

\ $P_{f}=0.20$
จ $P_{f}=0.30$

- $P_{f}=0.40$

४ $P_{f}=0.50$

(b)

$$
\begin{aligned}
& P_{f}=0.30 \\
& P_{f}=0.40 \\
& \checkmark P_{f}=0.50
\end{aligned}
$$

(d)

FIGURE 12: $S-\lg \mathrm{N}_{\mathrm{p}}$ relationships of AASC with various degrees of frost damage for various failure probabilities.(a) $n=0$. (b) $n=50$. (c) $n=100$. (d) $n=150$.

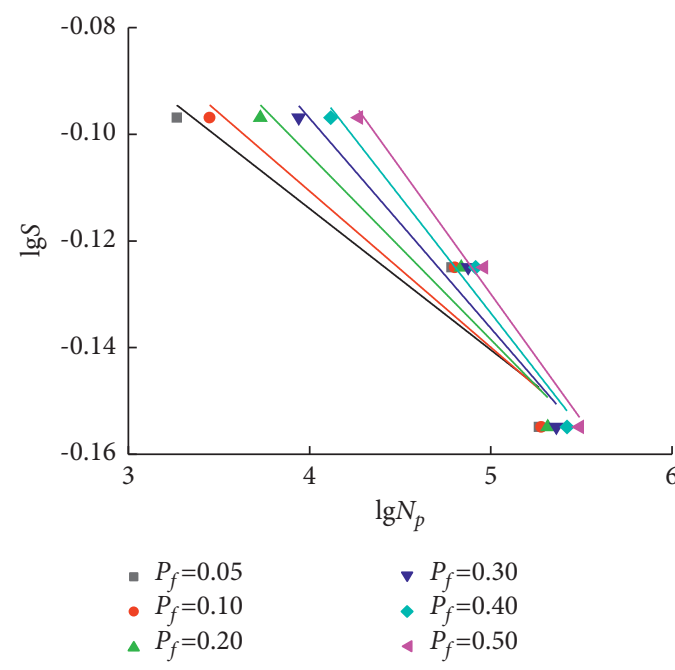

(a)

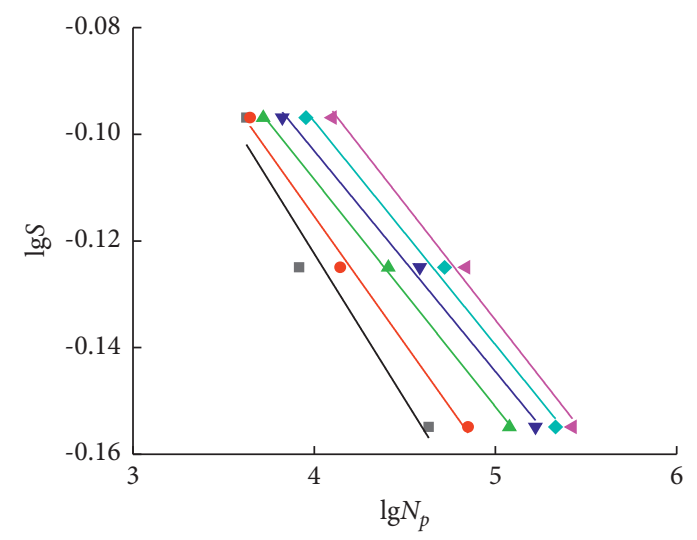

$$
\begin{aligned}
& \text { - } P_{f}=0.05 \\
& \text { - } P_{f}=0.10 \\
& \Delta P_{f}=0.20
\end{aligned}
$$

$\checkmark P_{f}=0.30$

- $P_{f}=0.40$

४ $P_{f}=0.50$

(b)

Figure 13: Continued. 


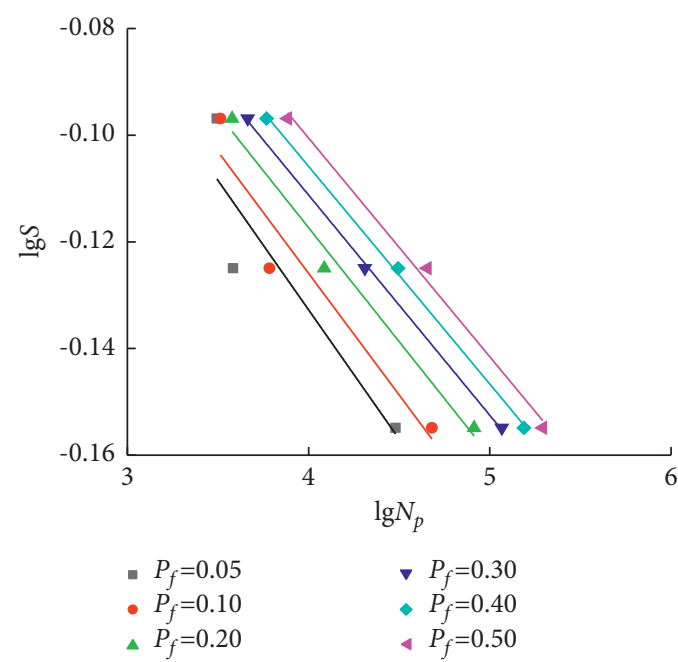

(c)

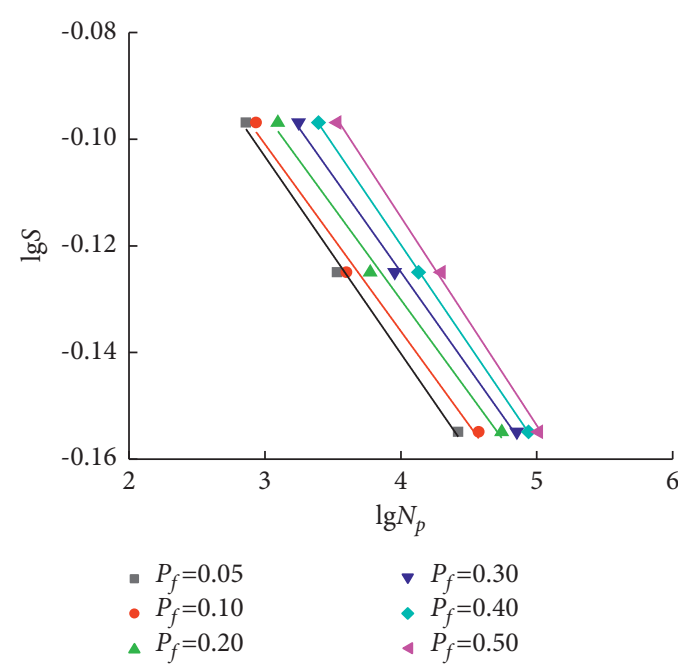

(d)

FIGURE 13: $\operatorname{lgS}-\lg \mathrm{N}_{\mathrm{p}}$ relationships of AASC with various degrees of frost damage for various failure probabilities. (a) $n=0$. (b) $n=50$. (c) $n=100$. (d) $n=150$.

TABLE 14: Single-logarithmic and double-logarithmic fatigue equations of AASC with various degrees of frost damage for various failure probabilities.

\begin{tabular}{|c|c|c|c|c|c|}
\hline$n$ & $P_{f}$ & Single-logarithmic fatigue equation & $R^{2}$ & Double-logarithmic fatigue equation & $R^{2}$ \\
\hline \multirow{6}{*}{0} & 0.05 & $S=0.9540-0.0459 \lg N$ & 0.9181 & $\lg S=0.0080-0.0265 \lg N$ & 0.9073 \\
\hline & 0.10 & $S=0.9790-0.0508 \lg N$ & 0.9293 & $\lg S=0.0065-0.0293 \lg N$ & 0.9192 \\
\hline & 0.20 & $S=1.0272-0.0599 \lg N$ & 0.9500 & $\lg S=0.0345-0.0346 \lg N$ & 0.9413 \\
\hline & 0.30 & $S=1.0719-0.0681 \lg N$ & 0.9680 & $\lg S=0.0604-0.0394 \lg N$ & 0.9609 \\
\hline & 0.40 & $S=1.1131-0.0754 \lg N$ & 0.9831 & $\lg S=0.0844-0.0436 \lg N$ & 0.9778 \\
\hline & 0.50 & $S=1.1503-0.0816 \lg N$ & 0.9941 & $\lg S=0.1062-0.0472 \lg N$ & 0.9908 \\
\hline \multirow{6}{*}{50} & 0.05 & $S=1.1308-0.0938 \lg N$ & 0.9444 & $\lg S=0.0963-0.0547 \lg N$ & 0.9529 \\
\hline & 0.10 & $S=1.0967-0.0826 \lg N$ & 0.9902 & $\lg S=0.0758-0.0478 \lg N$ & 0.9937 \\
\hline & 0.20 & $S=1.0737-0.0736 \lg N$ & 0.9999 & $\lg S=0.0621-0.0426 \lg N$ & 0.9992 \\
\hline & 0.30 & $S=1.0745-0.0714 \lg N$ & 0.9975 & $\lg S=0.0624-0.0414 \lg N$ & 0.9953 \\
\hline & 0.40 & $S=1.0877-0.0723 \lg N$ & 0.9958 & $\lg S=0.0700-0.0419 \lg N$ & 0.9930 \\
\hline & 0.50 & $S=1.1099-0.0752 \lg N$ & 0.9957 & $\lg S=0.0829-0.0435 \lg N$ & 0.9928 \\
\hline \multirow{6}{*}{100} & 0.05 & $S=1.0694-0.0829 \lg N$ & 0.8173 & $\lg S=0.0613-0.0485 \lg N$ & 0.8319 \\
\hline & 0.10 & $S=1.0623-0.0782 \lg N$ & 0.9122 & $\lg S=0.0566-0.0456 \lg N$ & 0.9228 \\
\hline & 0.20 & $S=1.0582-0.0735 \lg N$ & 0.9814 & $\lg S=0.0536-0.0427 \lg N$ & 0.9862 \\
\hline & 0.30 & $S=1.0594-0.0712 \lg N$ & 0.9979 & $\lg S=0.0540-0.0413 \lg N$ & 0.9993 \\
\hline & 0.40 & $S=1.0657-0.0704 \lg N$ & 0.9998 & $\lg S=0.0574-0.0408 \lg N$ & 0.9989 \\
\hline & 0.50 & $S=1.0764-0.0708 \lg N$ & 0.9969 & $\lg S=0.0635-0.0410 \lg N$ & 0.9945 \\
\hline \multirow{6}{*}{150} & 0.05 & $S=0.9792-0.0636 \lg N$ & 0.9933 & $\lg S=0.0076-0.0369 \lg N$ & 0.9961 \\
\hline & 0.10 & $S=0.9735-0.0604 \lg N$ & 0.9883 & $\lg S=0.0043-0.0351 \lg N$ & 0.9921 \\
\hline & 0.20 & $S=0.9826-0.0601 \lg N$ & 0.9899 & $\lg S=0.0096-0.0349 \lg N$ & 0.9933 \\
\hline & 0.30 & $S=0.9994-0.0620 \lg N$ & 0.9953 & $\lg S=0.0192-0.0360 \lg N$ & 0.9976 \\
\hline & 0.40 & $S=1.0185-0.0646 \lg N$ & 0.9993 & $\lg S=0.0302-0.0375 \lg N$ & 0.9999 \\
\hline & 0.50 & $S=1.0386-0.0674 \lg N$ & 0.9996 & $\lg S=0.0417-0.0391 \lg N$ & 0.9985 \\
\hline
\end{tabular}




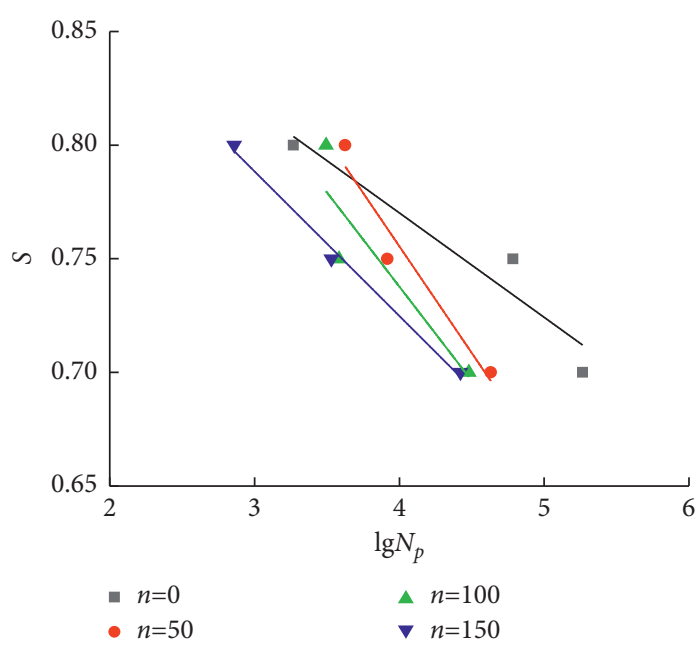

(a)

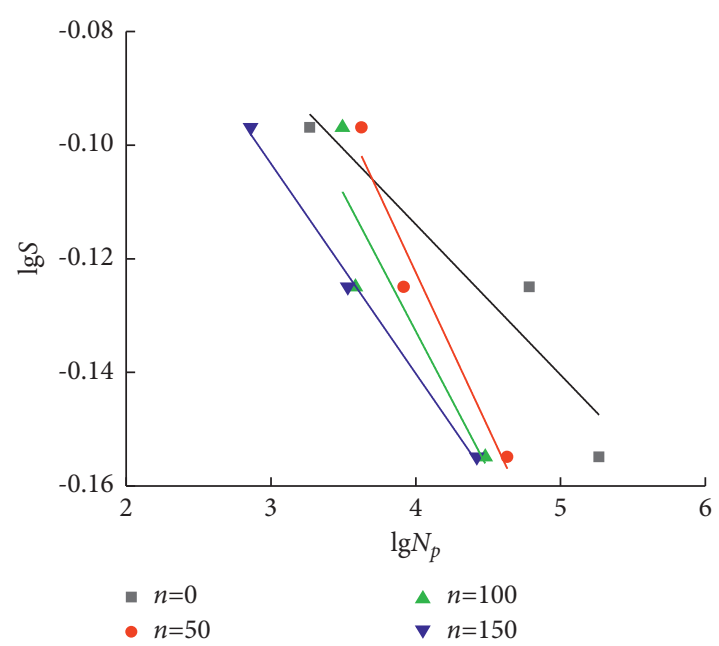

(b)

FIgURE 14: $S-\operatorname{lgN} N_{\mathrm{p}}$ and $\lg S-\operatorname{lgN} \mathrm{N}_{\mathrm{p}}$ relationships of AASC with various degrees of frost damage at $P_{f}=0.05$.

\section{Conclusions}

In this study, the influence of frost damage on the flexural fatigue life of AASC was studied through experiments, the influence of frost damage on the distribution of flexural fatigue life under different probability models was investigated, and the relevant distribution fitting tests and flexural fatigue reliability analysis were conducted. The main conclusions are summarized as follows:

(1) Frost damage clearly aggravated AASC flexural fatigue failure. With increasing degree of frost damage, the flexural fatigue life decreased substantially.

(2) In this study, the flexural fatigue lives of all the AASC samples were fit to two-parameter and three-parameter Weibull distributions, and $K-S$ test results were also analyzed. Combining the distribution test and $K-S$ test results, it is found that the three-parameter Weibull had a better fitting accuracy and results.

(3) The results of the two-parameter Weibull distribution obtained by the graphical method show that the flexural fatigue life of AASC is more discrete under high stresses, and at lower stresses, the dispersion in the flexural fatigue life increases with the aggravation of frost damage. The results of the three-parameter Weibull distribution obtained by the correlation coefficient method show that the dispersion in the flexural fatigue life of AASC gradually increases with the aggravation of frost damage.

(4) According to the two-parameter and three-parameter Weibull flexural fatigue reliability analysis, the curves of the undamaged and frost-damaged AASC with different failure probabilities at different stresses have good correlation. The ultimate strength of flexural fatigue with a three-parameter Weibull distribution shows that when $P_{f}$ is small, the flexural fatigue life is highly sensitive to freeze-thaw damage under high stresses.
(5) In this study, plain AASC samples were used, and the results are mainly applicable to the study and application of plain AASC components. The flexural fatigue equation of a reinforced AASC sample has not yet been established, but it is the next work to be done. However, it is clear from this study that frost damage has a significant impact on the flexural fatigue performance of AASC, and the reliability probability of parts that may suffer more serious frost damage should be improved during design and verification of the flexural fatigue life of AASC.

\section{Data Availability}

All data used to support the findings of this study are available from the corresponding author upon request.

\section{Conflicts of Interest}

The authors declare that they have no conflicts of interest.

\section{Acknowledgments}

This research was funded by Heilongjiang Traffic and Transportation Department (JTZD-20181826) and Fundamental Research Funds for the Central Universities (2572019CT01).

\section{References}

[1] X. Li, W. Wang, Z. Zhu, and K. Zheng, "Investigation on durability behaviour and optimization of concrete with tripleadmixtures subjected to freeze-thaw cycles in salt solution," Advances in Materials Science and Engineering, vol. 2021, Article ID 5572011, 16 pages, 2021.

[2] S. Luo, W. Liang, H. Wang, W. Wang, and R. Zou, "Durability evaluation of concrete with multiadmixtures under salt freeze-thaw cycles based on surface resistivity," Advances in Materials Science and Engineering, vol. 2021, Article ID 5567873, 18 pages, 2021. 
[3] P. Ganesh and A. Ramachandra Murthy, "Fatigue performance of damaged RC beams rehabilitated with GGBS based ultra high performance concrete," International Journal of Fatigue, vol. 138, Article ID 105707, 2020.

[4] M. H. Prashanth, P. Singh, and J. M. C. Kishen, "Role of longitudinal reinforcement on the behavior of under reinforced concrete beams subjected to fatigue loading," International Journal of Fatigue, vol. 125, pp. 271-290, 2019.

[5] Y. Tu, D. Liu, T. Wang, and L. Yuan, "Evaluation on later-age performance of concrete subjected to early-age freeze-thaw damage," Construction and Building Materials, vol. 270, Article ID 121491, 2021.

[6] A. Ebrahimi Besheli, K. Samimi, F. Moghadas Nejad, and E. Darvishan, "Improving concrete pavement performance in relation to combined effects of freeze-thaw cycles and de-icing salt," Construction and Building Materials, vol. 277, Article ID 122273, 2021.

[7] X. Yang, A. Shen, Y. Guo, S. Zhou, and T. He, "Deterioration mechanism of interface transition zone of concrete pavement under fatigue load and freeze-thaw coupling in cold climatic areas," Construction and Building Materials, vol. 160, pp. 588-597, 2018.

[8] Z. Li, T. Lu, Y. Chen, B. Wu, and G. Ye, "Prediction of the autogenous shrinkage and microcracking of alkali-activated slag and fly ash concrete," Cement and Concrete Composites, vol. 117, Article ID 103913, 2021.

[9] M. Tuyan, L. V. Zhang, and M. L. Nehdi, "Development of sustainable preplaced aggregate concrete with alkali-activated slag grout," Construction and Building Materials, vol. 263, Article ID 120227, 2020.

[10] M. Amran, G. Murali, N. H. A. Khalid et al., "Slag uses in making an ecofriendly and sustainable concrete: a review," Construction and Building Materials, vol. 272, Article ID 121942, 2021.

[11] Z. Jiao, Y. Wang, W. Zheng, and W. Huang, "Effect of the activator on the performance of alkali-activated slag mortars with pottery sand as fine aggregate," Construction and Building Materials, vol. 197, pp. 83-90, 2019.

[12] Z. Jiao, Y. Wang, W. Zheng, and W. Huang, "Effect of dosage of sodium carbonate on the strength and drying shrinkage of sodium hydroxide based alkali-activated slag paste," Construction and Building Materials, vol. 179, pp. 11-24, 2018.

[13] Z. Pan, Z. Tao, Y. F. Cao, R. Wuhrer, and T. Murphy, "Compressive strength and microstructure of alkali-activated fly ash/slag binders at high temperature," Cement and Concrete Composites, vol. 86, pp. 9-18, 2018.

[14] O. Burciaga-Díaz and J. I. Escalante-García, "Comparative performance of alkali activated slag/metakaolin cement pastes exposed to high temperatures," Cement and Concrete Composites, vol. 84, pp. 157-166, 2017.

[15] A. M. Rashad and G. M. F. Essa, "Effect of ceramic waste powder on alkali-activated slag pastes cured in hot weather after exposure to elevated temperature," Cement and Concrete Composites, vol. 111, Article ID 103617, 2020.

[16] Y. Fu, L. Cai, and W. Yonggen, "Freeze-thaw cycle test and damage mechanics models of alkali-activated slag concrete," Construction and Building Materials, vol. 25, no. 7, pp. 3144-3148, 2011.

[17] L. Cai, H. Wang, and Y. Fu, "Freeze-thaw resistance of alkalislag concrete based on response surface methodology," Construction and Building Materials, vol. 49, pp. 70-76, 2013.

[18] L. You, L. Jiang, and H. Chu, "Influence of carbonation on fatigue of concrete with high volume of ground granulated blast-furnace slag," Journal of Wuhan University of
Technology-Materials Science Edition, vol. 30, no. 2, pp. 361368, 2015.

[19] Y. Zhang, X. Wan, D. Hou, T. Zhao, and Y. Cui, "The effect of mechanical load on transport property and pore structure of alkali-activated slag concrete," Construction and Building Materials, vol. 189, pp. 397-408, 2018.

[20] J. L. Provis, Alkali-Activated Binders And Concretes: The Path To Standardization, University of Sheffield, Sheffield, England, 2013.

[21] F. Shahrajabian and K. Behfarnia, "The effects of nano particles on freeze and thaw resistance of alkali-activated slag concrete," Construction and Building Materials, vol. 176, pp. 172-178, 2018.

[22] H. Ma, H. Zhu, C. Wu, H. Chen, J. Sun, and J. Liu, "Study on compressive strength and durability of alkali-activated coal gangue-slag concrete and its mechanism," Powder Technology, vol. 368, pp. 112-124, 2020.

[23] V. S. Cândido, A. C. R. D. Silva, N. T. Simonassi, E. S. Lima, F. S. D. Luz, and S. N. Monteiro, "Mechanical and microstructural characterization of geopolymeric concrete subjected to fatigue," Journal of Materials Research and Technology, vol. 7, no. 4, pp. 566-570, 2018.

[24] A. Mohammadinia, A. Arulrajah, I. Phummiphan, S. Horpibulsuk, and M. Mirzababaei, "Flexural fatigue strength of demolition aggregates stabilized with alkali-activated calcium carbide residue," Construction and Building Materials, vol. 199, pp. 115-123, 2019.

[25] Standardization Administration of the People's Republic of China, GB/T 18046-2017: Ground Granulated Blast Furnace Slag Uesd for Cement, Mortar and Concrete, China Standards Press, Beijing, China, 2017.

[26] Ministry of Housing and Urban-Rural Development of People's Repoblic of China, GB/T 50081-2019: Standard for Test Methods of concrete Physical and Mechanical Properties, China Buliding Materials Industry Press, Beijing, China, 2019.

[27] R. Tepfers, "Tensile fatigue strength of plain concrete," Journal of the American Concrete Institute, vol. 76, pp. 919-933, 1979.

[28] B. H. Oh, "Fatigue analysis of plain concrete in flexure," Journal of Structural Engineering, vol. 112, no. 2, pp. 273-288, 1986.

[29] S. P. Singh and S. K. Kaushik, "Fatigue strength of steel fibre reinforced concrete in flexure," Cement and Concrete Composites, vol. 25, no. 7, pp. 779-786, 2003.

[30] Y. Mohammadi and S. K. Kaushik, "Flexural fatigue-life distributions of plain and fibrous concrete at various stress levels," Journal of Materials in Civil Engineering, vol. 17, no. 6, pp. 650-658, 2005.

[31] G. Kaur, S. P. Singh, and S. K. Kaushik, "Influence of mineral additions on flexural fatigue performance of steel fibre reinforced concrete," Materials and Structures, vol. 49, no. 10, pp. 4101-4111, 2016.

[32] J. Ríos, H. Cifuentes, R. Yu, and G. Ruiz, "Probabilistic flexural fatigue in plain and fiber-reinforced concrete," $M a$ terials, vol. 10, no. 7, p. 767, 2017.

[33] L.-X. Zhang, S.-Y. Wang, and Z.-D. Zhao, "Analysis of reliability confidence limits of fatigue damage strength of concrete," Gongcheng Lixue/Engineering Mech.vol. 21, pp. 139-143+132, 2004.

[34] M. Chen, H. Zhong, and M. Zhang, "Flexural fatigue behaviour of recycled tyre polymer fibre reinforced concrete," Cement and Concrete Composites, vol. 105, Article ID 103441, 2020.

[35] Z.-M. Ou and L. Sun, "Flexural fatigue-life reliability of frost-damaged concrete," Zhejiang Daxue Xuebao (Gongxue Ban)/Journal Zhejiang Univ. (Engineering Sci,vol. 51, pp. 1074-1081, 2017. 
[36] A. G. Graeff, K. Pilakoutas, K. Neocleous, and M. V. N. N. Peres, "Fatigue resistance and cracking mechanism of concrete pavements reinforced with recycled steel fibres recovered from post-consumer tyres," Engineering Structures, vol. 45, pp. 385-395, 2012.

[37] S. Goel and S. P. Singh, "Fatigue performance of plain and steel fibre reinforced self compacting concrete using S-N relationship," Engineering Structures, vol. 74, pp. 65-73, 2014. 